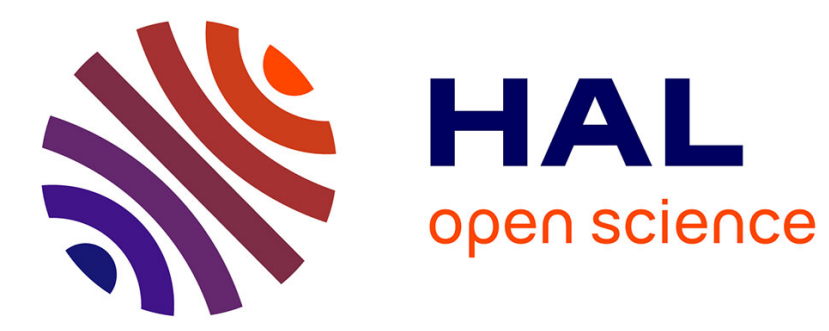

\title{
The common structure of the curves having a same Gauss word \\ Bruno Courcelle
}

\section{To cite this version:}

Bruno Courcelle. The common structure of the curves having a same Gauss word. A. Adamatzky. Automata, Computation, Universality, Springer, pp.1-37, 2015, Automata, Computation, Universality. hal-00879514

\section{HAL Id: hal-00879514 https://hal.science/hal-00879514}

Submitted on 4 Nov 2013

HAL is a multi-disciplinary open access archive for the deposit and dissemination of scientific research documents, whether they are published or not. The documents may come from teaching and research institutions in France or abroad, or from public or private research centers.
L'archive ouverte pluridisciplinaire HAL, est destinée au dépôt et à la diffusion de documents scientifiques de niveau recherche, publiés ou non, émanant des établissements d'enseignement et de recherche français ou étrangers, des laboratoires publics ou privés. 


\title{
The common structure of the curves having a same Gauss word
}

\author{
Bruno Courcelle \\ LaBRI, Bordeaux University and CNRS \\ Email : courcell@labri.fr
}

November 4, 2013

\begin{abstract}
Gauss words are finite sequences of letters associated with self-intersecting closed curves in the plane. (These curves have no "triple" selfintersection). These sequences encode the order of intersections on the curves. We characterize, up to homeomorphism, all curves having a given Gauss word. We extend this characterization to the $n$-tuples of closed curves having a given $n$-tuple of words, that we call a Gauss multiword . These words encode the self-intersections of the curves and their pairwise intersections. Our characterization uses a canonical decomposition of strongly connected graphs, called the atomic decomposition, that we have defined and studied in a previous article.
\end{abstract}

\section{Introduction}

Many geometric configurations can be represented by finite combinatorial objects, up to appropriate equivalence relations, like homeomorphism in the case of embeddings of graphs in surfaces. Gauss words are sequences of letters intended to describe the self-intersections of closed curves in the plane (with no triple intersection): each crossing is named by a letter, and a word with two occurrences of each letter is obtained by following the curve and writing the letter seen at each crossing. This definition raises the following questions:

(1) What are these words?

(2) Which curves can be uniquely reconstructed, up to homeomorphism, from the corresponding word?

(3) What is the common structure of all curves having a same associated word?

Gauss words are characterized in several articles by Lovasz and Marx [LM], Rosenstiehl [Ros] and de Fraysseix and Ossona de Mendez [FOM] to name a few. These works answer Question (1). Some of them are reviewed in the book by Godsil and Royle [GodRoy]. 

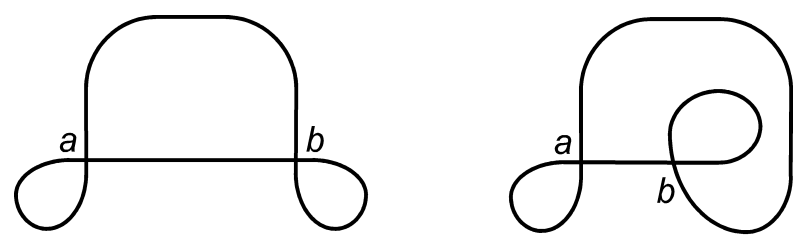

Figure 1: Curves with Gauss word $a a b b$.

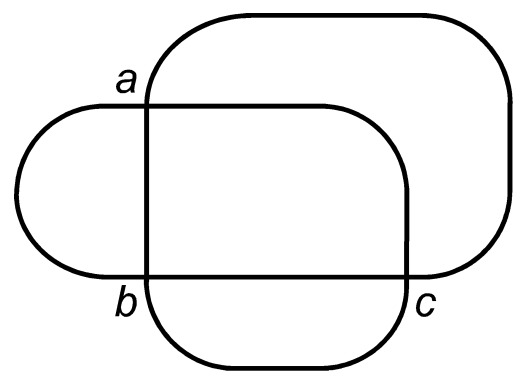

Figure 2: Curve with Gauss word $a b c a b c$

We will address Questions (2) and (3). A word is unambiguous if it characterizes a unique curve up to homeomorphism. Otherwise, it is ambiguous. Figure 1 shows two curves that are not related by any homeomorphism of the plane or of the sphere but yield the same ambiguous Gauss word $a a b b$. On the other hand, the word $a b c a b c$ is unambiguous and Figure 2 shows a (the) corresponding curve.

It is natural and convenient to extend the definitions and the corresponding questions to tuples of curves, described by tuples of words such that any letter has exactly two occurrences in one word or one occurrence in two of them. Figure 3 shows three curves with corresponding unambiguous Gauss multiword (abcd, aecf, bfde).

We will give in Proposition 23 a natural characterization of Gauss multiwords that yields (by means of Proposition 15) a linear-time recognition algorithm. This algorithm provides also a tuple of corresponding curves if there exists one. However, this proposition does not generalize the characterizations of [LM], [Ros], and [FOM].

For answering Questions (2) and (3), we will use several notions. First we observe that intersecting and self-intersecting closed curves without triple intersections are nothing but plane 4-regular graphs. We recall that a map is a graph equipped, at each vertex, with a circular order of edges around it. This order is called a rotation (see the book by Mohar and Thomassen [MT], Chapter 3). Every embedding of the graph in an oriented surface yields a map. 


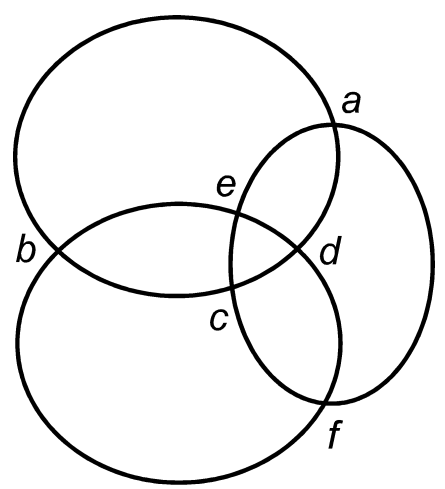

Figure 3: Three intersecting circles

Intersecting closed curves can be described up to homeomorphism by 4-regular planar maps (we omit here some technical details).

Second, we use a single combinatorial object, constructed from an $n$-tuple $W$ of words where each letter has two occurrences, from which the planar maps representing the $n$-tuples of curves with associated multiword $W$ can be defined. This object is a 4-regular graph with transitions, that is, equipped, at each vertex $v$, with a pairing of the half-edges, called darts, incident with $v$. We call it a t-graph. In its embeddings in surfaces, two paired darts must form a line that crosses the one formed by the two others incident darts. See Figure 5 for an example. (Pairings are represented by thickenings of certain darts. For example, edges $a$ and $b$ are paired at their common vertex, and so are $b$ and $c$ ). A $t$-graph is essentially a 4-regular map in which we forget the orientation of the surface around each vertex (we only retain how darts alternate around a vertex). Hence, a $t$-graph contains more information than the underlying graph and less than a map of this graph. A $t$-graph with an underlying planar graph may have no plane embedding that satisfies the "crossing condition" on pairs of opposite darts (see the right part of Figure 10). We will prove in Theorem 16 that, if the underlying graph of a planar $t$-graph is loop-free and 3-edge-connected, then this $t$-graph has a unique plane embedding, up to homeomorphism.

Third, we start our investigation with tuples of oriented curves (see Figure $4)$. The corresponding $t$-graphs are directed and each vertex has 2 incoming edges and 2 outgoing edges. We say that they are (2,2)-regular. Figure 4 shows the difference between oriented and nonorienting curves regarding the ambiguity of Gauss multiwords. It shows two plane embeddings of a $(2,2)$-regular $t$-graph associated with the Gauss multiword $(a b c d, b c, a d)$ that are not homeomorphic by any homeomorphism of the sphere. (To check this, just compare the directions of the edges incident with the two faces bordered by 4 edges). However, forgetting the directions of the edges yields two homeomorphic em- 

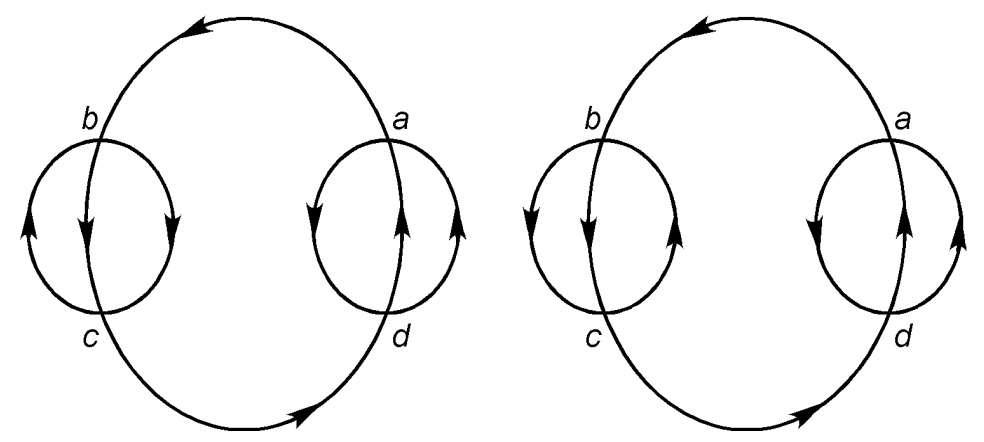

Figure 4: Two nonhomeomorphic embeddings of a same $t$-map.

beddings. Hence the multiword $(a b c d, b c, a d)$ is unambiguous for representing intersections of nonoriented closed curves, but it is ambiguous for representing intersections of oriented curves. It is actually easier to characterize the unambiguous Gauss multiwords representing oriented curves and we will start by this case.

Our key tool will be a canonical decomposition of strongly connected (directed) graphs that we have introduced in [CouAD] and called the atomic decomposition. Figure 9 in Section 3.2 below shows an atomic decomposition: the graphs $G_{1}, \ldots, G_{9}$ are undecomposable, they are the atoms of the decomposed graph.This decomposition works for the corresponding undirected graphs (they are the connected graphs without bridges), for $t$-graphs and for strongly connected maps. It is related to the canonical decomposition of a connected graph in 3-connected blocks due to Tutte [Tut]. From the atomic decomposition of the $t$-graph associated with a Gauss multiword $W$, we can describe all tuples of oriented curves (up to homeomorphism) whose associated Gauss multiword is $W$. Then, we extend this characterization to solve the original questions concerning nonoriented curves.

The article is organized as follows. Section 2 reviews definitions about graphs and maps. Section 3 reviews from [CouAD] the atomic decomposition of strongly connected graphs. Section 4 examines the decompositions of planar graphs and maps. Section 5 develops the applications to the curves in the plane described by given multiwords. Section 6 reviews open questions. For the reader's convenience, an appendix reviews the various equivalence and isomorphism notions used in this article.

\section{Definitions}

All graphs and related objects ( $t$-graphs, maps) will be finite. 
By saying that $\left(e_{1}, \ldots, e_{k}\right)$ is a circular sequence, we mean that it can also be specified as $\left(e_{i+1}, \ldots, e_{k}, e_{1}, \ldots, e_{i-1}\right)$ and that its properties and associated constructions do not depend on the initial element $e_{1}$. (See also Section 5.1.1).

\subsection{Graphs}

\subsubsection{Terminology and notation}

Terminology and notation are as in [CouAD]. A directed graph $G$ is defined as a triple $\left(V_{G}, E_{G}\right.$, vert $\left._{G}\right)$ consisting the set of vertices $V_{G}$, the set of edges $E_{G}$ (with $V_{G} \cap E_{G}=\emptyset$ ) and a mapping vert ${ }_{G}: E_{G} \rightarrow V_{G} \times V_{G}$ that defines incidences. If $\operatorname{vert}_{G}(e)=(x, y)$, we say that $x$ is the tail of $e$, denoted by $\alpha(e)$, that $y$ is its head, denoted by $\beta(e)$, we also write $e: x \rightarrow y$ and we say that $x$ and $y$ are the ends of $e$. If $G$ is undirected, then $\operatorname{vert}_{G}(e)$ is a set $\{x, y\}$ of one or two vertices, called the ends of $e$ and we write $e: x-y$. In both cases, $e$ is a loop if $x=y$.

We denote by $\operatorname{Und}(G)$ the undirected graph obtained from a directed one by taking as incidence function vert $\operatorname{Und}(G)_{(e)}:=\{x, y\}$ whenever $\operatorname{vert}_{G}(e)=(x, y)$. Note that we do not identify an edge with the pair or the set of its ends.

Walks and paths.

Let $G$ be a graph and $x, y \in V_{G}$. A walk from $x$ to $y$ is a sequence $\left(x_{0}, e_{1}, x_{1}, e_{2}, \ldots, e_{n}, x_{n}\right)$ such that $x_{0}, x_{1}, \ldots, x_{n} \in V_{G}, x_{0}=x, x_{n}=y, e_{1}, \ldots, e_{n}$ $\in E_{G}, e_{i}: x_{i-1} \rightarrow x_{i}\left(e_{i}: x_{i-1}-x_{i}\right.$ if $G$ is undirected) for each $i=1, \ldots, n$, and $e_{i} \neq e_{j}$ if $1 \leq i<j \leq n$. It is a path if we (also) have $x_{i} \neq x_{j}$ for $0 \leq i<j \leq n$, except possibly if $i=0$ and $j=n$. A walk is closed if $x_{0}=x_{n}$. A circuit is a closed path in a directed graph. A cycle is similar in an undirected graph (a cycle with two vertices consists of two parallel edges). An edge never occurs twice in a walk. A vertex never occurs twice in a path except if $x_{0}=x_{n}$. A walk $\left(x_{0}, e_{1}, x_{1}, e_{2}, \ldots, e_{n}, x_{n}\right)$ of a directed graph can be described without ambiguity by the sequence $\left(e_{1}, e_{2}, \ldots, e_{n}\right)$. A walk, a path or a circuit in a directed graph is said to be undirected if its edges can be traversed in any direction (i.e., with $e_{i}: x_{i-1} \rightarrow x_{i}$ or $e_{i}: x_{i} \rightarrow x_{i-1}$ in the above definition).

A directed graph is strongly connected if, for any two distinct vertices $x$ and $y$, there is path from $x$ to $y$. The class of strongly connected graphs is denoted by $\mathcal{S C}$.

\section{Subgraphs}

We write $G \subseteq H$ (resp. $G \subseteq_{i} H$ ) if $G$ is a subgraph (resp. an induced subgraph) of $H$. If $F \subseteq V_{G} \cup E_{G}$, then $G-F$ is the subgraph of $G$ obtained by deleting the edges and vertices in $F$ and the edges incident with a vertex in $F$. We write it $G-x$ if $F=\{x\}$. If $X \subseteq V_{G}$, we denote by $G[X]$ the graph $G-\left(V_{G}-X\right)$ : it is the induced subgraph of $G$ with vertex set $X$.

\section{Isomorphisms}


An isomorphism of $G=\left(V_{G}, E_{G}\right.$, vert $\left._{G}\right)$ to $G^{\prime}=\left(V_{G^{\prime}}, E_{G^{\prime}}\right.$, vert $\left._{G^{\prime}}\right)$ is a bijection $h: V_{G} \cup E_{G} \rightarrow V_{G^{\prime}} \cup E_{G^{\prime}}$ that maps vertices to vertices, edges to edges and preserves incidences, that is : vert $G^{\prime}(h(e))=(h(x), h(y))($ resp. $\{h(x), h(y)\})$ if $\operatorname{vert}_{G}(e)=(x, y)\left(\right.$ resp. $\left.\operatorname{vert}_{G}(e)=\{x, y\}\right)$. It is a v-isomorphism if $V_{G}=V_{G^{\prime}}$. In this case, one can consider $E_{G}$ and $E_{G^{\prime}}$ as different sets of names used to designate the edges of a graph with vertex set $V_{G}$. If $G^{\prime}=G$, we get the notions of automorphism and of v-automorphism. The graph of Figure 2 has several vautomorphisms. We denote by $G \cong G^{\prime}$ the existence of an isomorphism between $G$ and $G^{\prime}$.

Darts.

To discuss plane embeddings it will be useful to split a directed edge $e$ into two darts $e^{-}, e^{+}$with incidences defined by a function $\gamma$ such that $\gamma\left(e^{-}\right)=x$ and $\gamma\left(e^{+}\right)=y$ if $e: x \rightarrow y$. We denote by $D_{G}^{+}$the set of darts $e^{+}$, by $D_{G}^{-}$ the set of darts $e^{-}$and by $D_{G}$ the set $D_{G}^{+} \cup D_{G}^{-}$. It is clear that $D_{G}^{+} \subseteq D_{G^{\prime}}^{+}$and $D_{G}^{-} \subseteq D_{G^{\prime}}^{-}$if $G \subseteq G^{\prime}$. We will only use this notion for directed graphs.

\subsubsection{Graphs with transitions}

Definition 1 : (2,2)-regular graph.

A $(2,2)$-regular graph is a directed, 4-regular graph, each vertex of which has 2 incoming edges and 2 outgoing edges. We denote by $\mathcal{G}_{2,2}$ the class of connected (2,2)-regular graphs. Each such graph has an Eulerian tour, i.e., is covered by a closed walk (covered means that the walk goes through all edges). (See [Die], Section 1.8, where the proof given for undirected graphs extends easily to directed ones). It is thus strongly connected.

Definition 2 : Graph with transitions.

A $(2,2)$-regular graph with transitions is a pair $(G, \tau)$ consisting of a $(2,2)$ regular graph $G$ and a transition function defined as a bijection $\tau: D_{G}^{+} \rightarrow D_{G}^{-}$ such that $\gamma(\tau(d))=\gamma(d)$ for every $d \in D_{G}^{+}$. We say in this case that $d$ and $\tau(d)$ are opposite darts. For shortness sake, we will say that there is a transition from eto $f$ (or even that $(e, f)$ is a transition) if $\gamma\left(f^{-}\right)=\gamma\left(e^{+}\right)$and $\tau\left(e^{+}\right)=f^{-}$. If $H=(G, \tau)$, we let $\operatorname{Graph}(H)$ denote $G, V_{H}$ denotes $V_{G}$ and similarly for other items. A t-graph is a connected (2,2)-regular graph with transitions. We denote by $\mathcal{G}_{2,2}^{t}$ the class of $t$-graphs.

An isomorphism or a $v$-isomorphism of $t$-graphs must respect transitions in an obvious way. (An appendix reviews the different notions of isomorphism and equivalence relation used in this article.)

Definition 3 : Straight walk

A walk $\left(x_{0}, e_{1}, x_{1}, e_{2}, \ldots, e_{n}, x_{n}\right)$ in a $(2,2)$-regular graph with transitions is straight if it is not closed and $\tau\left(e_{i}^{+}\right)=e_{i+1}^{-}$for each $i=1, \ldots, n-1$ or if it is closed and, in addition to this condition, $\tau\left(e_{n}^{+}\right)=e_{1}^{-}$. Every $t$-graph is the 


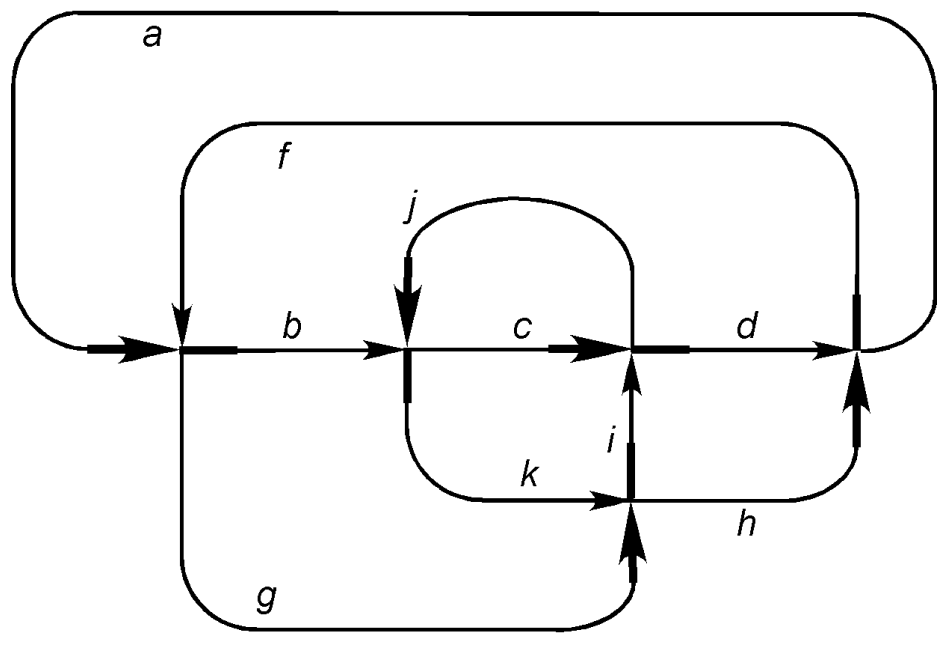

Figure 5: A planar $t$-map.

union of a set of pairwise edge-disjoint closed straight walks, in a unique way.

Figure 5 shows a (plane embedding of a) $t$-graph. The transition function is represented by thickening some darts: two bold darts are related by the transition, and so are two light darts. For example $\tau\left(a^{+}\right)=b^{-}$and $\tau\left(f^{+}\right)=g^{-}$. This graph is covered by the closed straight walks $(a, b, c, d)$ and $(f, g, i, j, k, h)$. Its Eulerian tour $(a, b, c, d, f, g, i, j, k, h)$ is not straight.

\subsection{Maps}

Maps are combinatorial objects that represent embeddings of connected graphs in oriented surfaces, up to orientation preserving homeomorphisms. We review the classical definitions (cf. [MT], Chapter 3 for detailed definitions), and we introduce some new notions.

If $\mathcal{E}$ is an embedding of a graph $G$ in a surface, we denote by $\mathcal{E}(u)$ the point representing a vertex $u$, by $\mathcal{E}(e)$ the line segment representing an edge eand by $\mathcal{E}(W)$ the union of the segments representing the edges of a walk $W$. As usual, we call plane an embedding of a graph in the sphere, and we define a homeomorphism of plane embeddings as a homeomorphism of the sphere that maps an embedding onto the other.

Definition 4: Map 

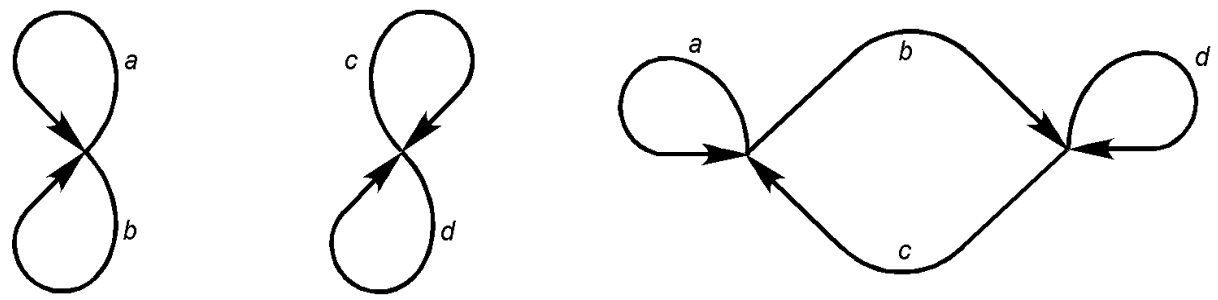

Figure 6: Three planar maps $M, N, P \in \mathcal{M}_{2,2}$

A map is a pair $M=(G, \rho)$ consisting of a connected and directed graph Gand a bijection $\rho: D_{G} \rightarrow D_{G}$ such that, for every $\operatorname{din} D_{G}$, the set $\left\{\rho^{i}(d) \mid i \geq\right.$ $0\}$ is the set of darts incident with $\gamma(d)$. This bijection is called the rotation of $M$. We denote $G$ by $\operatorname{Graph}(M)$ and $D_{G}$ by $D_{M}$.

From an embedding $\mathcal{E}$ of a connected and directed graph $G$ in an orientable surface, we get a map $(G, \rho)$ by letting $\rho(d)$ be the dart following $d$ in the circular order, "around the vertex $\gamma(d)$ " (and according to the orientation of the surface) of the darts incident with $\gamma(d)$. For any two embeddings of $G$ in the sphere with same associated map, there is an orientation preserving homeomorphism of the sphere that maps $\mathcal{E}$ to $\mathcal{E}^{\prime}$. (See [MT], Theorem 3.2.4 for the proof, and a more general statement concerning orientable surfaces.). A map is planar if it is associated with a plane embedding, i.e., an embedding in the sphere.

If $\mathcal{E}$ is a plane embedding of a connected and directed graph $G$ with map $M=(G, \rho)$ then $M^{-1}:=\left(G, \rho^{-1}\right)$ is the symmetric map of $M$ : it corresponds to an embedding $\mathcal{E}^{\prime}$ of $G$ that is homeomorphic to $\mathcal{E}$, with a reversal of orientations. We say that two maps $M$ and $M^{\prime}$ are equivalent if $M^{\prime}=M$ or $M^{\prime}=M^{-1}$. (They have the same underlying graph).

We denote respectively by $\mathcal{M S C}$ and $\mathcal{M}_{2,2}$ the classes of maps of graphs in $\mathcal{S C}$ and in $\mathcal{G}_{2,2}$.

Definition 5: Transitions defined from rotations.

Let $M=(G, \rho)$ be a map in $\mathcal{M}_{2,2}$. It is a $t$-map if the mapping $\tau$ defined by $\tau(d):=\rho^{2}(d)$ for $d \in D_{G}^{+}$is a transition function. The associated $t$-graph is $\operatorname{Graph}^{t}(M):=(G, \tau)$. We let $\mathcal{M}_{2,2}^{t}$ denote the class of $t$-maps. Two $t$-maps Mand Nare t-equivalent if $\operatorname{Graph}^{t}(M)=\operatorname{Graph}^{t}(N)$, which we denote by $M \sim^{t} N$. Clearly, $M \sim^{t} M^{-1}$. Figure 4 shows two $t$-maps $M$ and $N$ that are $t$-equivalent but not equivalent: they are different but $N \neq M^{-1}$. (Another similar example can be obtained from Figure 1).

In Figure 6, the planar map $M$ at the left is a $t$-map because $\rho^{2}\left(a^{+}\right)=b^{-}$and $\rho^{2}\left(b^{+}\right)=a^{-}$, hence $\rho^{2}$ is a transition function. The map $N$ in the middle is not because $\rho^{2}\left(c^{+}\right)=d^{+}$. (We will denote by 8 the corresponding graph in $\mathcal{G}_{2,2}$ ). The rightmost map $P$ is not either because $\rho^{2}\left(d^{+}\right)=b^{+}$. The planar map of Figure 5 is a $t$-map. 
Definition 6 : Planar t-graph

A plane embedding of a $t$-graph Grespects the transition if the corresponding map $M$ is such that $\operatorname{Graph}^{t}(M)=G$.A $t$-graph $G$ is planar if it has a plane embedding, hence, if and only if it is $\operatorname{Graph}^{t}(M)$ for some planar $t$-map $M$.

We denote by $\mathcal{P S C}, \mathcal{P} \mathcal{G}_{2,2}, \mathcal{P} \mathcal{G}_{2,2}^{t}, \mathcal{P} \mathcal{M S C}, \mathcal{P} \mathcal{M}_{2,2}$ and $\mathcal{P} \mathcal{M}_{2,2}^{t}$ the classes of graphs, $t$-graphs and maps that belong respectively to $\mathcal{S C}, \mathcal{G}_{2,2}, \mathcal{G}_{2,2}^{t}, \mathcal{M S C}$, $\mathcal{M}_{2,2}$ and $\mathcal{M}_{2,2}^{t}$ and are planar. These classes of graphs and maps, are related by inclusions and by the mappings Graphand Graph $^{t}$.We have in particular :

$$
\begin{aligned}
\mathcal{M}_{2,2} & \supseteq \mathcal{M}_{2,2}^{t} \\
\downarrow & \\
\mathcal{G}_{2,2} & \leftarrow \mathcal{G}_{2,2}^{t} .
\end{aligned}
$$

This square diagram shows that:

$$
\operatorname{Graph}(M)=\operatorname{Graph}\left(\operatorname{Graph}^{t}(M)\right) \text { for every } M \in \mathcal{M}_{2,2}^{t} .
$$

These relations and facts extend to planar graphs and maps. Hence, we have the similar square diagram with analogous meaning:

$$
\begin{array}{ccc}
\mathcal{P} \mathcal{M}_{2,2} & \supseteq & \mathcal{P} \mathcal{M}_{2,2}^{t} \\
\downarrow & & \downarrow \\
\mathcal{P} \mathcal{G}_{2,2} & \leftarrow & \mathcal{P} \mathcal{G}_{2,2}^{t}
\end{array}
$$

The symmetrization mapping $M \mapsto M^{-1}$ preserves each of the classes $\mathcal{M S C}$, $\mathcal{M}_{2,2}, \mathcal{M}_{2,2}^{t}, \mathcal{P} \mathcal{M S C}, \mathcal{P} \mathcal{M}_{2,2}, \mathcal{P} \mathcal{M}_{2,2}^{t}$.

\section{Atoms of graphs and maps}

A canonical decomposition of strongly connected graphs into so-called atoms is defined and studied in [CouAD]. It is applicable in a straightforward manner to maps. We review some definitions and show that they are applicable to $t$-graphs and to the graphs, $t$-graphs and maps of the classes of Definition 6 .

\subsection{Circular composition of directed graphs and maps}

We define an operation that composes directed graphs and maps. We will actually use it mainly to decompose these objects.

Definition 7 : Circular composition.

We let $G_{1}, G_{2}$ be disjoint directed graphs and $e_{i} \in E_{G_{i}}$ for $i=1,2$. We define $G_{1} \boxplus_{e_{1}, e_{2}} G_{2}$ as the graph $H$ such that $V_{H}:=V_{G_{1}} \cup V_{G_{2}}, E_{H}:=E_{G_{1}} \cup E_{G_{2}}$ and the incidence function vert $_{H}$ is defined as follows: 

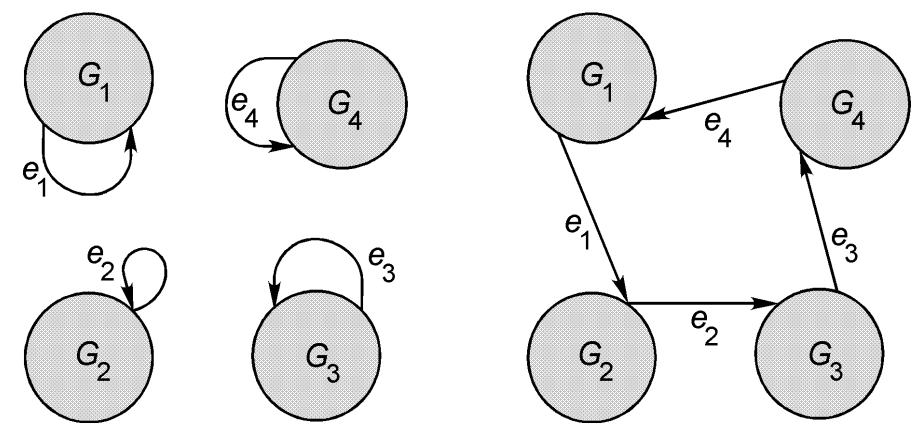

Figure 7: $G$ (to the right) is the circular composition of $G_{1}, \ldots, G_{4}$.

$$
\begin{aligned}
& \operatorname{vert}_{H}\left(e_{1}\right):=\left(\alpha\left(e_{1}\right), \beta\left(e_{2}\right)\right), \\
& \operatorname{vert}_{H}\left(e_{2}\right):=\left(\alpha\left(e_{2}\right), \beta\left(e_{1}\right)\right), \\
& \operatorname{vert}_{H}(e):=\operatorname{vert}_{G_{i}}(e) \text { if } e \in E_{G_{i}}-\left\{e_{i}\right\}, i=1,2 .
\end{aligned}
$$

We call $G_{1} \boxplus_{e_{1}, e_{2}} G_{2}$ a circular composition of $G_{1}$ and $G_{2}$. If $G_{1}, \ldots, G_{k}$ are pairwise disjoint and $e_{i}$ is an edge of $G_{i}$ for each $i$, we let:

$$
\begin{aligned}
& \left.\boxplus_{e_{1}, \ldots, e_{k}}\left(G_{1}, \ldots, G_{k}\right):=\left(\ldots\left(G_{1} \boxplus_{e_{1}, e_{2}} G_{2}\right) \boxplus_{e_{2}, e_{3}} G_{3}\right) \ldots\right) \boxplus_{e_{k-1}, e_{k}} G_{k} \\
& =\boxplus_{e_{1}, \ldots, e_{k-1}}\left(G_{1}, \ldots, G_{k-1}\right) \boxplus_{e_{k-1}, e_{k}} G_{k} .
\end{aligned}
$$

Here are examples. We let $G, H, K$ be the respective graphs of the maps $M, N, P$ of Figure 6. We have $K=G \boxplus_{b, c} H$. The left part of Figure 7 shows graphs $G_{1}, \ldots, G_{4}$ with distinguished edges $e_{1}, \ldots, e_{4}$ (note that $e_{2}$ is a loop). The right part shows $\boxplus_{e_{1}, \ldots, e_{4}}\left(G_{1}, G_{2}, G_{3}, G_{4}\right)$. This figure explains the terminology.

We have introduced and studied circular composition in [CouAD]. In the present article we will mainly use its "binary" version $G_{1} \boxplus_{e_{1}, e_{2}} G_{2}$. The following properties are easy to check from definitions.

Proposition 8 : Let $G_{1}, G_{2}, G_{3}$ be pairwise disjoint directed graphs and $e_{i} \in E_{G_{i}}$ for $i=1,2,3$. We have the following equalities (in each case, both handsides are defined):

(1) $G_{1} \boxplus_{e_{1}, e_{2}} G_{2}=G_{2} \boxplus_{e_{2}, e_{1}} G_{1}$,

(2) $\left(G_{1} \boxplus_{e_{1}, e_{2}} G_{2}\right) \boxplus_{e_{2}, e_{3}} G_{3}=G_{1} \boxplus_{e_{1}, e_{3}}\left(G_{2} \boxplus_{e_{2}, e_{3}} G_{3}\right)$,

(3) $\left(G_{1} \boxplus_{e_{1}, e_{2}} G_{2}\right) \boxplus_{f, e_{3}} G_{3}=G_{1} \boxplus_{e_{1}, e_{2}}\left(G_{2} \boxplus_{f, e_{3}} G_{3}\right)$ if $f \in E_{G_{2}}-\left\{e_{2}\right\}$. 
From (1) and (2) we get the following circular associativity :

$\left(G_{1} \boxplus_{e_{1}, e_{2}} G_{2}\right) \boxplus_{e_{2}, e_{3}} G_{3}=\left(G_{2} \boxplus_{e_{2}, e_{3}} G_{3}\right) \boxplus_{e_{3}, e_{1}} G_{1}$.

The graph $H=\boxplus_{e_{1}, \ldots, e_{4}}\left(G_{1}, G_{2}, G_{3}, G_{4}\right)$ at the right of Figure 7 can be expressed by $\left(G_{1} \boxplus_{e_{1}, e_{2}} G_{2}\right) \boxplus_{e_{2}, e_{4}}\left(G_{3} \boxplus_{e_{3}, e_{4}} G_{4}\right)$.

It is easy to check that $G_{1} \boxplus_{e_{1}, e_{2}} G_{2}$ is strongly connected if $G_{1}$ and $G_{2}$ are so. However, the connectedness of $G_{1}$ and $G_{2}$ does not imply that of $G_{1} \boxplus_{e_{1}, e_{2}} G_{2}$ : just take $G_{1}=e_{1}$ and $G_{2}=e_{2}$.

Definition 9: Circular composition of t-graphs and maps.

(a) We recall that $\mathcal{G}_{2,2} \subseteq \mathcal{S C}$. If $G_{1}$ and $G_{2}$ belong to $\mathcal{G}_{2,2}$, then so does any circular composition $G_{1} \boxplus_{e_{1}, e_{2}} G_{2}$. If they are disjoint $t$-graphs (i.e., if they belong to $\mathcal{G}_{2,2}^{t}$ ), then, we define $H=G_{1} \boxplus_{e_{1}, e_{2}} G_{2}$ as the $t$-graph with vertices and edges as in Definition 7 for graphs and transition function $\tau_{H}$ defined by:

$$
\begin{aligned}
& \tau_{H}\left(e_{1}^{+}\right):=\tau_{G_{2}}\left(e_{2}^{+}\right), \\
& \tau_{H}\left(e_{2}^{+}\right):=\tau_{G_{1}}\left(e_{1}^{+}\right), \\
& \tau_{H}(d):=\tau_{G_{i}}(d) \text { if } d \in D_{G_{i}}^{+}-\left\{e_{i}^{+}\right\}, i=1,2 .
\end{aligned}
$$

Hence, $H$ belongs to $\mathcal{G}_{2,2}^{t}$ because it is strongly connected and the degree conditions at each vertex are satisfied.

(b) Similarly, for pairwise disjoint strongly connected maps $M_{1}$ and $M_{2}$, we define a map $N=M_{1} \boxplus_{e_{1}, e_{2}} M_{2}$ with underlying graph $H=\operatorname{Graph}\left(M_{1}\right) \boxplus_{e_{1}, e_{2}}$ $\operatorname{Graph}\left(M_{2}\right)$ and rotation $\rho_{N}$ defined as follows:

$$
\begin{aligned}
& \rho_{N}\left(e_{1}^{+}\right):=\rho_{M_{2}}\left(e_{2}^{+}\right), \\
& \rho_{N}\left(e_{2}^{+}\right):=\rho_{M_{1}}\left(e_{1}^{+}\right), \\
& \rho_{N}(d):=e_{1}^{+} \text {if } d \in D_{M_{2}}-\left\{e_{2}^{+}\right\} \text {and } \rho_{M_{2}}(d)=e_{2}^{+}, \\
& \rho_{N}(d):=e_{2}^{+} \text {if } d \in D_{M_{1}}-\left\{e_{1}^{+}\right\} \text {and } \rho_{M_{1}}(d)=e_{1}^{+}, \\
& \rho_{N}(d):=\rho_{M_{i}}(d) \text { if } d \in D_{G_{i}}, i=1,2 \text { and the above cases do not } \\
& \text { apply. }
\end{aligned}
$$

Then, $H$ is strongly connected and $N$ is a map. If furthermore $M_{1}$ and $M_{2}$ belong to $\mathcal{M}_{2,2}$ or $\mathcal{M}_{2,2}^{t}$, then $N$ belongs to the same class.

For the maps of Figure 6, we have the equality $P=M \boxplus_{b, c} N$. Figure 7 shows the circular composition of four planar maps represented in the plane, with distinguished edges drawn "on the outer face". Figure 8 shows the composition $G_{1} \boxplus_{e_{1}, e_{2}} G_{2}$ of two planar maps $G_{1}$ and $G_{2}$, represented similarly, but with $e_{2}$ not on the outer face. 

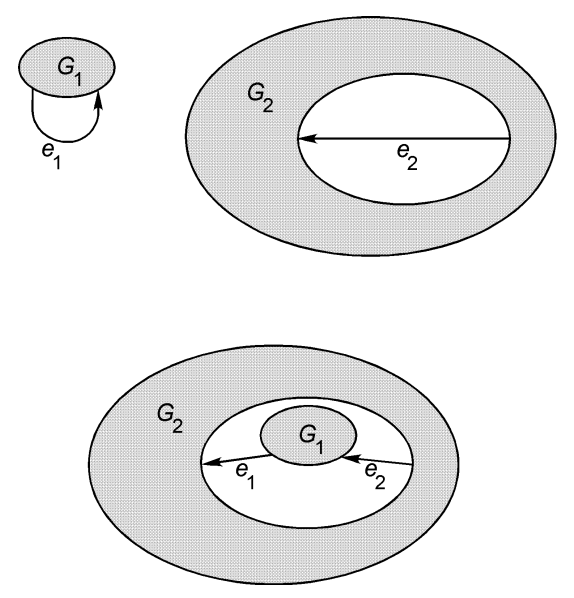

Figure 8: Composition of planar maps

The equational properties of circular composition stated in Proposition 8 are also valid for $t$-graphs and maps.

In Definitions 7 and 9, circular composition is a partial operation because of the disjointness condition on the arguments. We will use it to decompose given maps and graphs. That is, for given $H$, we will try to find graphs (or maps) $G_{1}$ and $G_{2}$ such that $H=G_{1} \boxplus_{e_{1}, e_{2}} G_{2}$. Hence, the disjointness condition does not raise any difficulty.

\subsection{Terms defining graphs and maps.}

Definition 10: Atoms and terms.

An atom is a strongly connected graph $G$ that cannot be expressed as $G_{1} \boxplus_{e, f}$ $G_{2}$, equivalently, that has no two edges $e$ and $f$ such that $G-\{e, f\}$ is not connected. The latter condition means that $G$ is 3-edge connected, hence that any two vertices are linked by 3 edge-disjoint undirected paths (by a theorem by Menger, cf. [Die] Theorem 3.3.6).

A $t$-atom (resp. an atomic map or $t$-map) is a $t$-graph (resp. a map or $t$-map) that cannot be expressed as the circular composition of two $t$-graphs or maps. If the context makes things clear, we will call them also atoms. (By Proposition 13 below they are the $t$-graphs, maps or $t$-maps whose underlying graphs are atoms.)

The graph $\operatorname{Graph}(M)=\operatorname{Graph}(N)$ where $M, N$ are at the left of Figure 6 and the graph of Figure 5 are atoms.

Let $\mathcal{A}$ be a set of pairwise disjoint graphs, $t$-graphs or maps included in one of the classes of Definition 6. A term over $\mathcal{A}$ is a term $t$ built with cicular 
composition and elements of $\mathcal{A}$ used as constants, each of them having at most one occurrence in the term. It defines a graph, a $t$-graph or a map, depending on the types of the elements of $\mathcal{A}$. We denote this object by $\operatorname{val}(t)$ : it is the value of $t$. We also say that $t$ defines $\operatorname{val}(t)$. We say that $t$ uses $\mathcal{A}$ if each element of $\mathcal{A}$ has one (and only one) occurrence in $t$.

Every strongly connected graph, $t$-graph or strongly connected map $G$ is defined by a term over atoms (of the corresponding type): if it is defined by a term $t$ using $G_{1}, \ldots, G_{k}$ such that $G_{i}$ is not an atom, then $G_{i}=H \boxplus_{e, f} K$ and $G_{i}$ can be replaced in $t$ by $H \boxplus_{e, f} K$. Proposition 8 shows that different terms (even over atoms) can have the same value. We will say that two such terms are equivalent. For example, the following two terms define the graph shown in Figure 9:

$$
\begin{aligned}
& t=\boxplus_{e_{1}, e_{2}, e_{3}}\left(G_{1},\left[\left(G_{2} \boxplus_{h_{2}, h_{7}} G_{7}\right) \boxplus_{f_{2}, f_{4}}\left(\boxplus_{g_{4}, g_{5}, g_{6}}\left(G_{4}, G_{5}, G_{6}\right)\right)\right], G_{3}\right) \\
& t^{\prime}=G_{7} \boxplus_{h_{7}, h_{2}}\left[\boxplus_{e_{1}, e_{2}, e_{3}}\left(G_{1}, G_{2}, G_{3}\right) \boxplus_{f_{2}, f_{4}}\left(\boxplus_{g_{4}, g_{5}, g_{6}}\left(G_{4}, G_{5}, G_{6}\right)\right)\right] .
\end{aligned}
$$

Theorem 11 ([CouAD], Theorem 17) : Every strongly connected graph $G$ is defined by a term over atoms. Any two terms defining it use the same set of atoms.

The set of atoms arising in the second assertion is called the set of atoms of $G$. We proved in [CouAD] that the different terms over atoms that define a strongly connected graph $G$ can be represented by a single (canonical) structure called the atomic decomposition of $G$. It is a directed cactus, i.e., a loop-free strongly connected graph whose 2-connected components are circuits. Each vertex of this cactus is an atom of $G$ (Figure 9 shows an example). In the present article, we will only use terms over atoms and the unicity property of the previous theorem.

Theorem 12 ([CouAD], Theorem 27) : There exists a linear-time algorithm that constructs, for every strongly connected graph, a term over atoms that defines it.

By the following proposition, these theorems extend to the classes of graphs, $t$-graphs and maps of Definition 6 .

Proposition 13 : (1) If $G, H_{1}, H_{2}$ are directed graphs such that $G=$ $H_{1} \boxplus_{e_{1}, e_{2}} H_{2}$, then $G$ is strongly connected (resp. is in $\mathcal{G}_{2,2}$ ) if and only if $H_{1}$ and $H_{2}$ are strongly connected (resp. are in $\mathcal{G}_{2,2}$ ). Furthermore, if $G, H_{1}, H_{2}$ are strongly connected, then $G$ is planar if and only if $H_{1}$ and $H_{2}$ are planar.

(2) If $G$ is a $t$-graph such that $\operatorname{Graph}(G)=H_{1} \boxplus_{e_{1}, e_{2}} H_{2}$ for some directed graphs $H_{1}$ and $H_{2}$, then there are unique transition functions $\tau_{1}, \tau_{2}$ such that $G=G_{1} \boxplus_{e_{1}, e_{2}} G_{2}$ and for each $i, G_{i}=\left(H_{i}, \tau_{i}\right) \in \mathcal{G}_{2,2}^{t}$. Furthermore, $G$ is planar if and only if $G_{1}$ and $G_{2}$ are planar. 
(3) If $M$ is a strongly connected map (resp. a map in $\mathcal{M}_{2,2}$ or a $t$-map) such that $\operatorname{Graph}(M)=H_{1} \boxplus_{e_{1}, e_{2}} H_{2}$ for some directed graphs $H_{1}$ and $H_{2}$, then there are unique rotations $\rho_{1}, \rho_{2}$ such that $M=N_{1} \boxplus_{e_{1}, e_{2}} N_{2}$ where, for each $i, N_{i}=\left(H_{i}, \rho_{i}\right)$ is a map (resp. is in $\mathcal{M}_{2,2}$ or in $\left.\mathcal{M}_{2,2}^{t}\right)$. Furthermore, $M$ is planar and strongly connected if and only if $N_{1}$ and $N_{2}$ are planar and strongly connected.

Proof: (1) Let $G, H_{1}, H_{2}$ be directed graphs such that $G=H_{1} \boxplus_{e_{1}, e_{2}} H_{2}$. Every closed walk of $G$ that goes through $e_{1}$ must also go through $e_{2}$. It follows then that $G$ is strongly connected if and only if $H_{1}$ and $H_{2}$ are so. The "local" conditions on edges for membership in $\mathcal{G}_{2,2}$ are easy to check.

Let $G, H_{1}, H_{2}$ be strongly connected. If $H_{1}$ and $H_{2}$ are planar, then a plane embedding of $G$ can be built from plane embeddings of $H_{1}$ and $H_{2}$ (cf. Figures 7 and 8). Conversely, assume that $G=H_{1} \boxplus_{e_{1}, e_{2}} H_{2}$ is planar, with plane embedding $\mathcal{E}$. Let $P$ be a path in $G$ from $x=\alpha\left(e_{1}\right)$ to $y=\beta\left(e_{2}\right)$ ( $y$ is a vertex of $H_{2}$ ) that goes through $e_{1}$, some edges of $H_{2}$ and finally $e_{2}$. Then, $\mathcal{E}(P)$ links $\mathcal{E}(x)$ to $\mathcal{E}(y)$. We obtain in this way a plane embedding of $H_{1}$. That $H_{2}$ is planar is proved similarly. (If we only assume that $H_{1} \boxplus_{e_{1}, e_{2}} H_{2}$ is planar and $H_{2}$ is connected, then $H_{1}$ may not be planar.)

(2) Let $G$ be a $t$-graph such that $\operatorname{Graph}(G)=H_{1} \boxplus_{e_{1}, e_{2}} H_{2}$ for some $H_{1}, H_{2}$ in $\mathcal{G}_{2,2}$. The graphs $\operatorname{Graph}(G), H_{1}$ and $H_{2}$ are strongly connected. The existence of unique transition functions $\tau_{1}, \tau_{2}$ such that $G=\left(H_{1}, \tau_{1}\right) \boxplus_{e_{1}, e_{2}}\left(H_{2}, \tau_{2}\right)$ and $\left(H_{1}, \tau_{1}\right),\left(H_{2}, \tau_{2}\right) \in \mathcal{G}_{2,2}^{t}$ follows easily. From plane embeddings of $\left(H_{1}, \tau_{2}\right)$ and $\left(H_{2}, \tau_{2}\right)$, one can build a plane embedding of $G$. The converse is proved as in (1).

(3) Let $M$ be a strongly connected map such that $\operatorname{Graph}(M)=H_{1} \boxplus_{e_{1}, e_{2}} H_{2}$ for some graphs $H_{1}, H_{2}$. These graphs must be strongly connected. The proof goes as for (2).

Theorem 14: Let $\mathcal{C}$ be any one of the classes $\mathcal{P S C}, \mathcal{G}_{2,2}, \mathcal{P} \mathcal{G}_{2,2}, \mathcal{G}_{2,2}^{t}, \mathcal{P} \mathcal{G}_{2,2}^{t}$, $\mathcal{M S C}, \mathcal{P} \mathcal{M S C}, \mathcal{M}_{2,2}, \mathcal{P} \mathcal{M}_{2,2}, \mathcal{M}_{2,2}^{t}$ or $\mathcal{P} \mathcal{M}_{2,2}^{t}$. Every element of $\mathcal{C}$ is defined by a term over atoms of $\mathcal{C}$. Any two terms defining it use the same set of atoms. There exists a linear-time algorithm that constructs, for every element of $\mathcal{C}$, a term over atoms that defines it.

Proof: The proof is the same for all cases. Let us do it for, say, $\mathcal{C}=\mathcal{P} \mathcal{G}_{2,2}^{t}$.

Let $G \in \mathcal{C}$. The graph $H=\operatorname{Graph}(G)$ is strongly connected, hence defined by a term $t$ using the atoms $H_{1}, \ldots, H_{k}$. By means of an induction on the structure of $t$, Proposition 13 entails the existence of unique transition functions $\tau_{1}, \ldots, \tau_{k}$ such that $\left(H_{i}, \tau_{i}\right) \in \mathcal{C}$ and $G$ is defined by a term using $\left(H_{1}, \tau_{1}\right), \ldots,\left(H_{k}, \tau_{k}\right)$. Since $H_{1}, \ldots, H_{k}$ are atoms in $\mathcal{S C},\left(H_{1}, \tau_{1}\right), \ldots,\left(H_{k}, \tau_{k}\right)$ are atoms in $\mathcal{C}$.

For proving the second assertion, consider a term $s^{\prime}$ using the atoms $G_{1}^{\prime}, \ldots, G_{\ell}^{\prime}$ in $\mathcal{C}$ that defines $G$. We get from it a term $s$ using the atoms $\operatorname{Graph}\left(G_{1}^{\prime}\right), \ldots$, $\operatorname{Graph}\left(G_{\ell}^{\prime}\right)$ in $\mathcal{S C}$ that defines $H$. Hence, $k=\ell$ and $\left\{H_{1}, \ldots, H_{k}\right\}=\left\{\operatorname{Graph}\left(G_{1}^{\prime}\right)\right.$, 


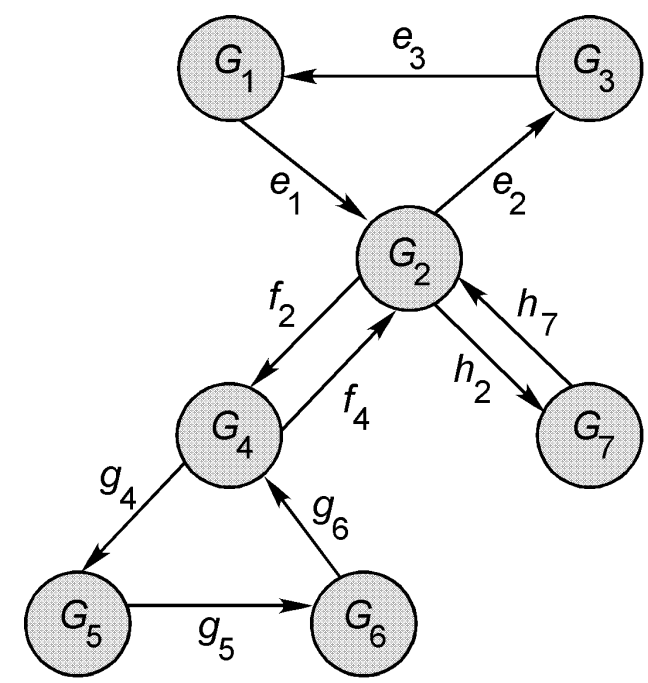

Figure 9: The graph definied by term $t$.
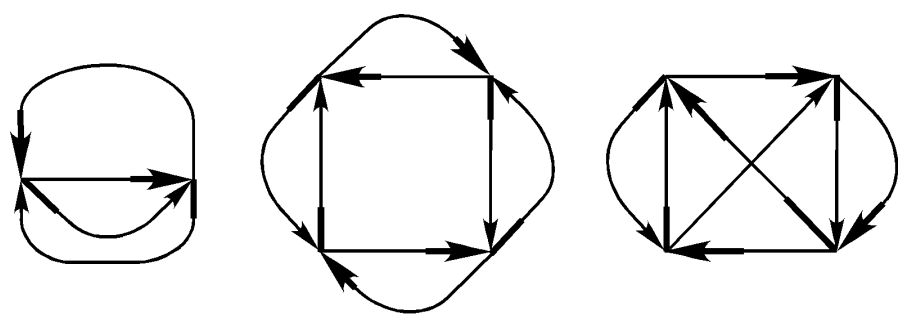

Figure 10: Three atomic $t$-maps.

..., Graph $\left.\left(G_{k}^{\prime}\right)\right\}$. Since in Proposition 13(2) the transition functions $\tau_{1}, \tau_{2}$ are uniquely defined, we have $\left\{\left(H_{1}, \tau_{1}\right), \ldots,\left(H_{k}, \tau_{k}\right)\right\}=\left\{G_{1}^{\prime}, \ldots, G_{k}^{\prime}\right\}$.

The algorithm of Theorem 12 can construct the atoms $H_{1}, \ldots, H_{k}$ of $H$. By Definition 9 , one can get the transition functions $\tau_{1}, \ldots, \tau_{k}$ in linear-time. $\square$

Figure 5 shows an atomic planar $t$-map. Figure 6 shows an atomic planar $t$-map $M$, and an atomic planar map $N$ that is not a $t$-map. The first two drawings of Figure 10 represent atoms that are planar considered as graphs, $t$-graphs or maps. The third one shows an atom that is planar as a graph but is not a planar $t$-graph or map.

We will need the following proposition of independent interest. A loop $e$ of $G \in \mathcal{G}_{2,2}^{t}$ has a transition to itself if $\tau\left(e^{+}\right)=e^{-}$. 
Proposition 15: There is a linear-time algorithm that checks if a $t$-graph is planar, and constructs if possible a plane embedding.

Proof : Let $G \in \mathcal{G}_{2,2}^{t}$. If $\operatorname{Graph}(G)$ has 1 or 2 vertices, we check if $G$ is planar by looking at its transition function.

Otherwise, we first eliminate the loops. Let $G$ have a loop $e$ at vertex $u$. If $e$ has a transition to itself, then $G$ is not planar. Otherwise, we remove $e$, we fuse the two remaining edges incident with $u$ (hence, we also remove $u$ ) and we repeat these removals until there is no more loop or we get a $t$-graph with 1 or 2 vertices. We obtain a $t$-graph $G^{\prime} \in \mathcal{G}_{2,2}^{t}$ that is planar if and only if $G$ is. If $G^{\prime}$ has 1 or 2 vertices, we conclude by looking at the transition function.

Otherwise $G^{\prime}$ has at least three vertices and no loop, and we construct a graph $H$ as follows:

(1) We subdivide each edge $e$ into two edges by putting a new vertex $x_{e}$ in its "middle".

(2) Let $u$ be any vertex. For any two edges $e$ and $f \neq e$ incident with $u$ such that there is no transition at $u$ between $e$ and $f$, we add an edge between $x_{e}$ and $x_{f}$. We say that we make $G^{\prime}$ rigid at $u$. We define $H$ by making $G^{\prime}$ rigid at all its vertices.

It is clear that $H$ is planar if and only if $G^{\prime}$ is, and that every plane embedding of $H$ yields one of the $t$-graph $G^{\prime}$ (that respects its transitions), from which we get easily one of the given $t$-graph $G$.

The constructions of $G^{\prime}$ from $G$ and of $H$ from $G^{\prime}$ can be done in time $O\left(\left|V_{G}\right|+\left|E_{G}\right|\right)$; the size of $H$ defined as $\left|V_{H}\right|+\left|E_{H}\right|$ is also $O\left(\left|V_{G}\right|+\left|E_{G}\right|\right)$. The classical linear-time planarity test applied to $H$ gives the answer. This algorithm constructs plane embeddings of $H, G^{\prime}$ and $G$ when they exist.

\section{Planar $t$-graphs and $t$-maps}

In order to answer our initial questions about curves in the plane, we now focus our attention on planar $t$-graphs and $t$-maps.

We first recall a basic fact. Let $M$ be a map and $G=\operatorname{Graph}(M)$. If $P$ is an undirected path in $G$ from $x$ to $y$ (cf. Section 2.1 for definitions) whose edge sequence is $\left(e_{1}, \ldots, e_{k}\right)$, if $d$ is the dart of $e_{1}$ such that $\gamma(d)=x$ and $d^{\prime}$ is that of $e_{k}$ such that $\gamma\left(d^{\prime}\right)=y$, they we say $d$ and $d^{\prime}$ are the end darts of $P$.

Let $M$ be planar, and let $P_{1}, P_{2}, P_{3}$ be vertex-disjoint undirected paths from $x$ to $y$ with respective end darts $d_{1}, d_{2}, d_{3}$ that are incident with $x$ and $d_{1}^{\prime}, d_{2}^{\prime}, d_{3}^{\prime}$ incident with $y$. If the circular order of $d_{1}, d_{2}, d_{3}$ (around $\left.x\right)$ is $\left(d_{1}, d_{2}, d_{3}\right)$, then that of $d_{1}^{\prime}, d_{2}^{\prime}, d_{3}^{\prime}$ around $y$ is $\left(d_{3}^{\prime}, d_{2}^{\prime}, d_{1}^{\prime}\right)$ (see [Cou00]). It follows that the circular order of $d_{1}, d_{2}, d_{3}$ determines that of $d_{1}^{\prime}, d_{2}^{\prime}, d_{3}^{\prime}$.

We recall from Definition 5 that two maps $M$ and $N$ in $\mathcal{M}_{2,2}^{t}$ are $t$-equivalent if and only if $\operatorname{Graph}^{t}(M)=\operatorname{Graph}^{t}(N)$, and that they are equivalent if and only 
if $M=N$ or $M=N^{-1}$. Two equivalent maps are $t$-equivalent. For atoms, we have the following converse.

Theorem 16 : If two maps $M$ and $N$ in $\mathcal{P} \mathcal{M}_{2,2}^{t}$ are $t$-equivalent and $\operatorname{Graph}(M)$ is an atom, then, they are equivalent. A planar $t$-atom has a unique plane embedding.

As atoms are 3-edge connected, this result is similar to the fact that a 3connected planar graph has a unique plane embedding ([Die, MT]). In the following definition and proofs, we will consider undirected paths and walks in directed graphs, that we will simply call paths and walks.

Definition 17 : Let $G \in \mathcal{G}_{2,2}^{t}$ and let $P$ and $Q$ be two edge-disjoint walks from $x$ to $y \neq x$. We say that they cross at a vertex $u$ belonging to $P$ and $Q$ if $u \neq x, u \neq y$ and the two darts of the edges of $P$ that are incident with $u$ are opposite (and so must be the two darts of the edges of $Q$ that are incident with $u$ ). If $P$ and $Q$ are vertex-disjoint (except for $x$ and $y$ ), they do not cross (at any vertex).

Similarly, we say that $P$ crosses itself at $u$ if it is of the form $\left(\ldots, e, u, e^{\prime}\right.$, $\left.\ldots, f, u, f^{\prime}, \ldots\right)$ where the darts of $e$ and $e^{\prime}$ incident with $u$ are opposite (and so must be the darts of $f$ and $f^{\prime}$ incident with $u$ ).

Lemma 18 : Let $G \in \mathcal{G}_{2,2}^{t}$ be atomic. Between any two distinct vertices, there are three edge-disjoint paths that pairwise do not cross.

Proof: If $G=\mathbf{8}$ the assertion is trivially true. Otherwise, since $G$ is atomic, there are three edge-disjoint paths between any two distinct vertices (cf. Definition 10). Let $P, Q, R$ be a triple of edge-disjoint paths from $x$ to $y \neq x$ and let $p$ be the total number of vertices at which they cross. Let $u$ be a vertex at which $P$ and $Q$ cross. Since $G$ is (2,2)-regular, $u$ is not on $R$. Then $P=P_{1} P_{2}$ and $Q=Q_{1} Q_{2}$ such that the paths $P_{1}$ and $Q_{1}$ go from $x$ to $u$, the paths $P_{2}$ and $Q_{2}$ go from $u$ to $y$. Then, $P_{1} Q_{2}$ and $Q_{1} P_{2}$ are two walks from $x$ to $y$. They are still edge-disjoint (and also edge-disjoint with $R$ ). The total number of self-crossings of $P_{1} Q_{2}$ and $Q_{1} P_{2}$ and of crossings between $P_{1} Q_{2}, Q_{1} P_{2}$ and $R$ is $p-1$. If $P_{1} Q_{2}$ is not a path, we can shorten it into a path $P^{\prime}$ from $x$ to $y$ by removing some closed subwalks. Similarly, we can shorten $Q_{1} P_{2}$ into a path $Q^{\prime}$ from $x$ to $y$. We get a triple $P^{\prime}, Q^{\prime}, R$ of edge-disjoint paths from $x$ to $y$ whose total number of crossings is at most $p-1$ (because the shortenings of $P_{1} Q_{2}$ and $Q_{1} P_{2}$ into $P^{\prime}$ and $Q^{\prime}$ can only reduce the number of crossings). By repeating this step, we get three edge-disjoint paths from $x$ to $y$ that pairwise do not cross.

Proof of Theorem 16 : Let $M, N \in \mathcal{P} \mathcal{M}_{2,2}^{t}$ be $t$-equivalent such that $\operatorname{Graph}(M)$ is an atom. If $\operatorname{Graph}(M)=\mathbf{8}$, then the result is clear. Otherwise, $G=\operatorname{Graph}(M)$ is loop-free (because if it has a loop, it has two edges $e, f$ such that $G=H_{1} \boxplus_{e, f} H_{2}$ and is not an atom). 
Let $x \in V_{G}$. If the rotations $\rho_{M}$ and $\rho_{N}$ are not the same on the darts incident with $x$, we have $\rho_{M}=\rho_{N^{-1}}$ at $x$ because $\operatorname{Graph}^{t}(M)=\operatorname{Graph}^{t}(N)$ and so, there are only two rotations around a vertex that yield (cf. Definition $5)$ a given transition function. We now replace $N$ by $N^{-1}$, and then $\rho_{M}$ and $\rho_{N}$ are the same on the darts incident with $x$.

We now prove that for every vertex $y \neq x$, the rotations $\rho_{M}$ and $\rho_{N}$ are the same on the darts incident with $y$. We first consider the case where $x$ and $y$ are linked by three vertex-disjoint paths. Then, since the circular order of the corresponding three end darts incident with $x$ is the same in $M$ and in $N$, the same holds for the circular order of the three darts around $y$ because of the three vertex-disjoint paths. The position of the fourth dart incident with $y$ among the first three is determined by the transition. Since we have $\operatorname{Graph}^{t}(M)=\operatorname{Graph}^{t}(N)$, the rotations $\rho_{M}$ and $\rho_{N}$ are the same on the four darts incident with $y$.

We now consider the general case, where $x$ and $y$ are linked by three edgedisjoint paths $P, Q, R$ that are not vertex-disjoint. By Lemma 18, we can assume that they pairwise do not cross. Let $W$ be the set of vertices different from $x$ and $y$ that belong to two of these paths. We add vertices to subdivide each edge of $G$ that has an end in $W$ and we also add edges between these new vertices, so as to make $G$ rigid at all vertices of $W$ (this notion is defined in the proof of Proposition 15). We obtain a graph $H$. Thanks to the newly added edges and because $P, Q, R$ do not cross pairwise, $x$ and $y$ are linked in $H$ by three vertex-disjoint paths $P^{\prime}, Q^{\prime}, R^{\prime}$ that avoid the vertices of $W$. We identify in a obvious way the darts of $G$ incident with $x$ and $y$ with the corresponding ones in $H$.

Consider plane embeddings $\mathcal{E}$ and $\mathcal{E}^{\prime}$ of $M$ and $N$ that respect the transition relations. By adding some line segments to $\mathcal{E}$, one can make it into a plane embedding $\mathcal{E}_{1}$ of $H$, and similarly, one can make $\mathcal{E}^{\prime}$ into a plane embedding $\mathcal{E}_{1}^{\prime}$ of $H$. The circular orders of darts around $x$ are the same in $M$ and $N$, and thus also in $\mathcal{E}_{1}$ and $\mathcal{E}_{1}^{\prime}$. Since we have three vertex-disjoint paths $P^{\prime}, Q^{\prime}, R^{\prime}$ between $x$ and $y$, the corresponding circular orders of their end darts around $y$ are the same in $\mathcal{E}_{1}$ and $\mathcal{E}_{1}^{\prime}$, hence in $M$ and in $N$. As observed above, and since $M$ and $N$ are $t$-equivalent, the rotations $\rho_{M}$ and $\rho_{N}$ are the same on the four darts incident with $y$.

We obtain that $M=N$, which gives the result. (We recall that the initial map $N$ may have been replaced by $N^{-1}$ at the beginning.)

Our objective is now to describe all maps in $\mathcal{P M}_{2,2}^{t}$ that are $t$-equivalent to a given map. For doing that we will use terms over atoms.

Lemma 19 : Let $M, M^{\prime}, N, N^{\prime}, P, P^{\prime} \in \mathcal{P} \mathcal{M}_{2,2}^{t}$ be such that $M \sim^{t} M^{\prime}, M=$ $N \boxplus_{e, f} P, M^{\prime}=N^{\prime} \boxplus_{e, f} P^{\prime}, \operatorname{Graph}(N)=\operatorname{Graph}\left(N^{\prime}\right)$ and $\operatorname{Graph}(P)=\operatorname{Graph}\left(P^{\prime}\right)$. Then $N \sim^{t} N^{\prime}$ and $P \sim^{t} P^{\prime}$.

Proof: The transition functions of $\operatorname{Graph}^{t}(N)$ and $\operatorname{Graph}^{t}(M)$ are related as follows: 


$$
\begin{aligned}
& \tau_{\operatorname{Graph}^{t}(N)}\left(e^{+}\right)=\tau_{\operatorname{Graph}^{t}(M)}\left(f^{+}\right), \\
& \tau_{\operatorname{Graph}^{t}(N)}(d)=\tau_{\operatorname{Graph}^{t}(M)}(d) \text { for every } d \in D_{\operatorname{Graph}^{t}(N)}^{+}-\left\{e^{+}\right\},
\end{aligned}
$$

and similarly for $M^{\prime}$ and $N^{\prime}$. Since $\operatorname{Graph}^{t}(M)=\operatorname{Graph}^{t}\left(M^{\prime}\right)$, we have $\operatorname{Graph}^{t}(N)=\operatorname{Graph}^{t}\left(N^{\prime}\right)$ and similarly, $\operatorname{Graph}^{t}(P)=\operatorname{Graph}^{t}\left(P^{\prime}\right)$. Hence $N \sim^{t}$ $N^{\prime}$ and $P \sim^{t} P^{\prime}$.

Let $t$ be a term over atomic maps. We will write terms by using atomic maps, the operation $\boxplus$ and also the symmetrization ${ }^{-1}$ that is well-defined on maps. We define as follows a set $\nabla(t)$ of such terms:

$$
\begin{aligned}
& \nabla(t):=\left\{t, t^{-1}\right\} \text { if } t \text { is an atomic map, } \\
& \nabla(t):=\left\{s^{\prime} \boxplus_{e, f} s^{\prime \prime} \mid s^{\prime} \in \nabla\left(t^{\prime}\right), s^{\prime \prime} \in \nabla\left(t^{\prime \prime}\right)\right\} \text { if } t=t^{\prime} \boxplus_{e, f} t^{\prime \prime} .
\end{aligned}
$$

Since for maps $M$ and $N$ we have $\left(M \boxplus_{e, f} N\right)^{-1}=M^{-1} \boxplus_{e, f} N^{-1}$, we need not add the terms $\left(s^{\prime} \boxplus_{e, f} s^{\prime \prime}\right)^{-1}$ to $\nabla(t)$ in the second case. In other words, the operation $^{-1}$ can be used equivalently anywhere in a term or only on atoms.

Theorem 20 : Let $M \in \mathcal{P} \mathcal{M}_{2,2}^{t}$ be defined by a term $t$ over atomic $t$-maps. The maps that are $t$-equivalent to $M$ are those defined by the terms in $\nabla(t)$.

Proof: We use an induction on the structure of $t$. If $t$ is an atom, the result follows from Theorem 16 .

Otherwise, $t=t^{\prime} \boxplus_{e, f} t^{\prime \prime}$. Lemma 19 shows that the $t$-maps that are $t$ equivalent to $M$ are those of the form $N \boxplus_{e, f} P$ where $N \sim^{t} \operatorname{val}\left(t^{\prime}\right)$ and $P \sim^{t}$ $\operatorname{val}\left(t^{\prime \prime}\right)$. By induction, these maps $N$ and $P$ are those defined respectively by the terms in $\nabla\left(t^{\prime}\right)$ and in $\nabla\left(t^{\prime \prime}\right)$. Hence, the $t$-maps $t$-equivalent to $M$ are those defined by the terms in $\nabla(t)$.

Corollary 21 : Let $M \in \mathcal{P} \mathcal{M}_{2,2}^{t}$ be defined by a term $t$ using $p$ atomic maps. There are $2^{p-1}$ pairwise inequivalent maps in $\mathcal{P} \mathcal{M}_{2,2}^{t}$ for the $t$-graph $\operatorname{Graph}^{t}(M)$. One can determine them from $t$.

Proof: We first observe that for all $M$ and $N$ in $\mathcal{P} \mathcal{M}_{2,2}^{t}$, the $t$-maps $M \boxplus_{e, f} N$ and $M \boxplus_{e, f} N^{-1}$ are $t$-equivalent but not equal. To prove this, we let $P=$ $M \boxplus_{e, f} N$ and $P^{\prime}=M \boxplus_{e, f} N^{-1}$. Then, $\rho_{P}\left(e^{+}\right)=\rho_{N}\left(f^{+}\right), \rho_{P^{\prime}}\left(e^{+}\right)=\rho_{N^{-1}}\left(f^{+}\right)$ and $\rho_{N^{-1}}\left(f^{+}\right) \neq \rho_{N}\left(f^{+}\right)$because the head of $f$ has degree 4 in $N$. Hence, $P \neq P^{\prime}$. Furthermore, $P^{\prime-1}=\left(M \boxplus_{e, f} N^{-1}\right)^{-1}=M^{-1} \boxplus_{e, f} N$, hence $P \neq P^{\prime-1}$ by the same argument and $M \boxplus_{e, f} N$ and $M \boxplus_{e, f} N^{-1}$ are not equivalent either.

It follows that two distinct terms $t^{\prime}$ and $t^{\prime \prime}$ in $\nabla(t)$ define different (but $t$ equivalent) maps. The set $\nabla(t)$ contains $2^{p}$ terms that define $2^{p}$ maps. Each of these maps $Q$ is equivalent to a unique map $Q^{-1}$ in the same set, and $Q \neq$ $Q^{-1}$ because these maps are 4-regular. Hence, we obtain exactly $2^{p-1}$ pairwise inequivalent maps. By fixing one atom of a term $t$ and by replacing in all possible 
ways some of the other atoms $M$ by $M^{-1}$, we obtain $2^{p-1}$ terms that define the $2^{p-1}$ pairwise inequivalent maps in $\mathcal{P} \mathcal{M}_{2,2}^{t}$ for the $t$-graph $\operatorname{Graph}^{t}(M)$.

For an example, the multiword $W=(a b c d, b c, a d)$ is ambiguous (see the introduction or the next section for the definition) since it is associated with the two triples of curves of Figure 4 . The $t$-graph ${ }^{t} G r a(W)$ has two atoms, hence, has two inequivalent planar maps, shown in Figure 4.

Remark : The maps $M \boxplus_{e, f} N$ and $M \boxplus_{e, f} N^{-1}$ are not equivalent because $M$ and $N$ are 4-regular. For comparison, we have $P=P^{-1}$ if $P$ is the (unique) map of a path or a circuit. It follows that $M \boxplus_{e, f} N=M \boxplus_{e, f} N^{-1}$ if $N$ is the map of a circuit containing the edge $f$.

\section{Curves in the plane}

\subsection{Oriented curves}

\subsubsection{Definitions and basic facts}

We make precise some definitions sketched in the introduction.

\section{Circular sequences}

We denote by $V^{*}$ the set of possibly empty sequences of elements of a finite set $V$, also considered as words over $V$ (whence the term "Gauss word"). The empty sequence is denoted by $\varepsilon$. Two sequences $u$ and $v$ are conjugate, which we denote by $u \equiv v$, if $u=w_{1} w_{2}$ and $v=w_{2} w_{1}$ for some $w_{1}, w_{2} \in V^{*}$. This relation is an equivalence and its classes are the circular sequences of elements of $V$. Their set is denoted by $V^{\circledast}$. We denote by $\operatorname{Conj}(u)$ the set of sequences conjugate with $u$.

The reversal (or mirror image) of a sequence $u$ is denoted by $\widetilde{u}$. Two sequences $u$ and $v$ are reversal-conjugate, which we denote by $u \asymp v$, if $u \equiv v$ or $u \equiv \widetilde{v}$. This is also an equivalence relation. Its equivalence classes are the reversal-circular sequences over $V$ and their set is denoted by $V^{\odot}$. We will usually designate an element of $V^{\circledast}$ or $V^{\odot}$ by some sequence of the corresponding equivalence class.

A double occurrence multiword over $V$ is a tuple of circular sequences over $V$ where each element of $V$ has two occurrences (either two occurrences in one sequence or one occurrence in two sequences). We denote by $D O_{n}(V)$ the set of such $n$-tuples. If $X \subseteq V$ and $W \in D O_{n}(V)$, we denote by $W \uparrow X$ the multiword in $D O_{n}(X)$ obtained by removing from the sequences composing $W$ the elements not in $X$. If $W, W^{\prime} \in D O_{n}(V)$, we write $W \asymp W^{\prime}$ if, for each $i$, the $i$-th component of $W$ is $\asymp$-equivalent to the $i$-th component of $W^{\prime}$.

Curves in the plane 
Let $\mathcal{C}$ be a closed oriented curve in the plane, with finitely many selfintersections but no triple (or more complex) self-intersection. The term oriented means that $\mathcal{C}$ is given with a traversal direction. We let $V$ be the set of self-intersection points, or more precisely, a set of letters naming these points. By following the curve from some of these points according to its traversal direction, we get a circular sequence $w(\mathcal{C})$ of elements of $V$ where each element of $V$ has two occurrences (hence, $(w(\mathcal{C})) \in D O_{1}(V)$ ). If $\mathcal{C}$ has no self-intersection, i.e., if it is homeomorphic to a circle, then, $w(\mathcal{C})=\varepsilon$. The sequence $w(\mathcal{C})$ is circular because the starting point is arbitrary. If $\mathcal{C}$ is not oriented, then $w(\mathcal{C}) \in V^{\odot}$ because the traversal direction is also arbitrary. Such sequences are called Gauss words, and have been studied in many articles (e.g., [ChaWeb, FOM, LRS, LM, Ros]).

Some sequences, for instance $a b a b$, are not Gauss words as one checks easily. (But a curve yielding it can be drawn on the torus). The Gauss word abcabc represents the curve of Figure 2. A square, i.e., a sequence of the form uu (where $u$ is a nonempty sequence) is a Gauss word if and only if $u$ has odd length.

We wish to characterize the cases where $\mathcal{C}$ can be reconstructed from $w(\mathcal{C})$, in a unique way up to homeomorphism. This is not always the case: Figure 1 shows two curves that do not correspond via any homeomorphism of the sphere but have the same Gauss word aabb. We will characterize the Gauss words which correspond to a unique curve, where as usual, unicity is understood up to homeomorphism.

Tuples of curves and graphs.

We will consider more generally finite tuples of intersecting and self-intersecting closed curves on the plane. With such an $n$-tuple of oriented curves $\left(\mathcal{C}_{1}, \ldots, \mathcal{C}_{n}\right)$, we associate the $n$-tuple of circular sequences $W\left(\mathcal{C}_{1}, \ldots, \mathcal{C}_{n}\right)=\left(w\left(\mathcal{C}_{1}\right)\right.$, $\left.\ldots, w\left(\mathcal{C}_{n}\right)\right) \in D O_{n}(V)$ where $V$ is the set of intersection and self-intersection points, or rather a set of names for these points. We call $W\left(\mathcal{C}_{1}, \ldots, \mathcal{C}_{n}\right)$ a Gauss multiword. Examples are $(\varepsilon, \varepsilon, \varepsilon),(a b c d, b f d e, a e c f)$ and $(a b c d, a b c d)$. The first one corresponds to three disjoint circles, the second one corresponds to the three curves of Figure 3 and the third one to two ellipses with 4 intersections. This last example shows that a same sequence may occur twice in a multiword. The 2 -tuple $(a, a)$ represents two curves on the torus with a single intersection, but does not represent two curves on the plane, this is a consequence of the following proposition.

Proposition 22 : Each component of a Gauss multiword has even length. The number of letters that occur in any two components of a Gauss multiword is even.

Proof: It follows from the Jordan Curve Theorem (see [MT], Chapter 2) that any two closed curves without self-intersections (they are homeomorphic to circles) cross at an even number of points. Hence, the two assertions hold for the 
Gauss multiwords associated with curves without self-intersections. (They are the Gauss multiwords such that each letter occurs in two different components.)

We now prove the general case by induction on the total number of selfintersections of the curves $\mathcal{C}_{1}, \ldots, \mathcal{C}_{n}$ that define the considered multiword. We let $W=W\left(\mathcal{C}_{1}, \ldots, \mathcal{C}_{n}\right)$ and, without loss of generality, we assume that $\mathcal{C}_{1}$ has a selfintersection named $a$. Hence $w\left(\mathcal{C}_{1}\right) \equiv$ auav. One can delete the self-intersection $a$ by replacing $\mathcal{C}_{1}$ by $\mathcal{C}_{1}^{\prime}$ such that $w\left(\mathcal{C}_{1}^{\prime}\right)=u \widetilde{v}$. The two assertions hold for $W$ if they hold for $W\left(\mathcal{C}_{1}^{\prime}, \mathcal{C}_{2}, \ldots, \mathcal{C}_{n}\right)$. The result follows. $\square$

t-graphs from multiwords.

We will mainly consider connected tuples of curves $\left(\mathcal{C}_{1}, \ldots, \mathcal{C}_{n}\right)$, i.e., tuples whose union is a connected subset of the sphere (in which we define our "plane embeddings"). If $n>1$, each curve has intersections with other curves. Except if the tuple consists of one circle, the union of $\mathcal{C}_{1}, \ldots, \mathcal{C}_{n}$ is a plane embedding $\mathcal{E}$ of a (nonempty) $t$-graph $G=G\left(\mathcal{C}_{1}, \ldots, \mathcal{C}_{n}\right) \in \mathcal{P G}_{2,2}^{t}$ that we now define. We split each curve $\mathcal{C}_{i}$ into a union of consecutive segments $s_{i, 1}, s_{i, 2}, \ldots, s_{i, n_{i}}$ whose ends are the intersection and self-intersection points, and we let $V$ be (in bijection with) the set of ends of these segments. We define $G$ as follows:

its vertex set $V_{G}$ is $V$,

its edges are the pairs $(i, j)$ such that $1 \leq i \leq n$ and $1 \leq j \leq n_{i}$, vert $_{G}((i, j))$ is the pair $(v, w)$ such that the segment $s_{i, j}$ links $v$ to $w$,

the transition is defined by : $\tau_{G}\left((i, j)^{+}\right):=(i, j+1)^{-}$if $1 \leq j<n_{i}$, and $\tau_{G}\left(\left(i, n_{i}\right)^{+}\right):=(i, 1)^{-}$.

A plane embedding $\mathcal{E}$ of $G$ is defined by $\mathcal{E}((i, j)):=s_{i, j}$. It is clear that $G$ is a planar $t$-graph (in particular because $\left(\mathcal{C}_{1}, \ldots, \mathcal{C}_{n}\right)$ is assumed connected) and that $\mathcal{E}$ respects its transition (cf. Definition 6 ). This $t$-graph has $n$ closed straight walks $\omega_{1}, \ldots, \omega_{n}$ : the edges of $\omega_{i}$ are those of the form $(i, j)$ and $\mathcal{E}\left(\omega_{1}\right), \ldots$, $\mathcal{E}\left(\omega_{n}\right)$ are the curves $\mathcal{C}_{1}, \ldots, \mathcal{C}_{n}$.

For the example of Figure 3 with trigonometric orientation of the curve, the graph $G\left(\mathcal{C}_{1}, \mathcal{C}_{2}, \mathcal{C}_{3}\right)$ has vertices $\{a, b, \ldots, f\}$, edges $a \rightarrow b, b \rightarrow c, c \rightarrow d, \ldots, b \rightarrow f$, $f \rightarrow d, \ldots, a \rightarrow e, e \rightarrow c, \ldots, f \rightarrow a$ and transitions $(a \rightarrow b, b \rightarrow c),(b \rightarrow c, c \rightarrow d)$, $\ldots,(b \rightarrow f, f \rightarrow d), \ldots,(c \rightarrow f, f \rightarrow a)$.

We have a bijection between the edges of $G\left(\mathcal{C}_{1}, \ldots, \mathcal{C}_{n}\right)$ and the segments $s_{i, j}$ of the curves $\mathcal{C}_{1}, \ldots, \mathcal{C}_{n}$. Every other plane embedding $\mathcal{E}^{\prime}$ of $G\left(\mathcal{C}_{1}, \ldots, \mathcal{C}_{n}\right)$ yields a tuple of oriented curves $\left(\mathcal{C}_{1}^{\prime}, \ldots, \mathcal{C}_{n}^{\prime}\right)$ (with $\mathcal{C}_{i}^{\prime}=\mathcal{E}^{\prime}\left(\omega_{i}\right)$ ) such that $W\left(\mathcal{C}_{1}^{\prime}, \ldots, \mathcal{C}_{n}^{\prime}\right)=W\left(\mathcal{C}_{1}, \ldots, \mathcal{C}_{n}\right)$

We now show that the $t$-graph $G\left(\mathcal{C}_{1}, \ldots, \mathcal{C}_{n}\right)$ can be constructed from $W\left(\mathcal{C}_{1}, \ldots\right.$, $\left.\mathcal{C}_{n}\right)$

Definition 23 : 
(a) A multiword $W \in D O_{n}(V)$ is connected if there is no bipartition $\left(V_{1}, V_{2}\right)$ of $V$ (with $V_{1}, V_{2}$ possibly empty) such that some components of $W$ are in $V_{1}^{*}$ and others in $V_{2}^{*}$. This is equivalent to the property that the graph $\left(V, R_{W}\right)$ is connected where $R_{W}:=\left\{(a, b) / w a b w^{\prime}\right.$ or bwa is a factor of some component of $W$ for some words $\left.w, w^{\prime}\right\}$. (Hence $(\varepsilon)$ is connected but $\left(\varepsilon, w_{2}, \ldots, w_{n}\right)$ is not.)

If $L \subseteq V^{*}, u, v \in L$, we say that $u$ and $v$ are connected via $L$ if there exist $z_{1}, \ldots, z_{p}$ in $L$ such that $u=z_{1}, v=z_{p}$ and for each $i, z_{i}$ and $z_{i+1}$ have at least one letter in common. Then $W$ is connected if and only if any two distinct components are connected via its set of components. It is clear that $W\left(\mathcal{C}_{1}, \ldots, \mathcal{C}_{n}\right)$ is connected if and only if $\left(\mathcal{C}_{1}, \ldots, \mathcal{C}_{n}\right)$ is connected.

(b) A multiword $W=\left(w_{1}, w_{2}, \ldots, w_{n}\right) \in D O_{n}(V)$ is prime if it is connected, different from $(\varepsilon)$ and there is no bipartition $\left(V_{1}, V_{2}\right)$ of $V$ (with $V_{1}, V_{2}$ nonempty) and index $i$ such that $w_{i}$ is conjugate to a word in $V_{1}^{+} V_{2}^{+}$and $w_{j} \in V_{1}^{+} \cup V_{2}^{+}$ for every $j \neq i$. For example, $(a a)$ and $(a b a b)$ are prime whereas $a a b b$ is not. The multiword $(a b c d, a b c d)$ is prime whereas $(a b c d, a b, c d)$ is not. The prime multiword (abcd, abef, cdfe) corresponds to the curves of Figure 3.

(c) The t-graph associated with a connected multiword.

Let $W=\left(w_{1}, w_{2}, \ldots, w_{n}\right) \in D O_{n}(V)$ where each $w_{i}$ is nonempty and written $v_{i, 1} \ldots, v_{i, n_{i}}$ with $v_{i, j} \in V$. We assume that each letter of $V$ has occurrences in $W$. We define a $t$-graph ${ }^{t} G r a(W)$ as follows:

$$
\begin{aligned}
& V_{t} \operatorname{Gra}(W):=V, \\
& E_{t_{G r a}(W)}:=\left\{(i, j) \mid 1 \leq j \leq n_{i}\right\}, \\
& \operatorname{vert}_{t} \operatorname{Gra}(W)((i, j)):=\left(v_{i, j}, v_{i, j+1}\right) \text { if } j<n_{i} \text { and } \operatorname{vert}_{t} \operatorname{Gra}(W)\left(\left(i, n_{i}\right)\right):= \\
& \left(v_{i, n_{i}}, v_{i, 1}\right), \\
& \tau_{t} \operatorname{Gra}(W) \\
&
\end{aligned}
$$

If $W^{\prime}=\left(w_{1}^{\prime}, \ldots, w_{n}^{\prime}\right)$ where $w_{i}^{\prime} \equiv w_{i}$, then ${ }^{t} G r a\left(W^{\prime}\right)$ is v-isomorphic to ${ }^{t} \operatorname{Gra}(W)$ : some edges $(i, j)$ of ${ }^{t} G r a(W)$ are just mapped to $\left(i, j^{\prime}\right)$. Here is an example. For $W=(a b, a b c c)$, we have $v_{1,1}=v_{2,1}=a, v_{1,2}=v_{2,1}=b$, $v_{2,1}=v_{2,2}=c$. Then ${ }^{t} G r a(W)$ is the $t$-graph $G$ with vertices $a, b, c$, edges $(1,1): a \rightarrow b,(1,2): b \rightarrow a,(2,1): a \rightarrow b,(2,2): b \rightarrow c,(2,3): c \rightarrow c$ and $(2,4): c \rightarrow a$. If $W^{\prime}=(a b, c c a b)$, then ${ }^{t} G r a\left(W^{\prime}\right)$ is v-isomorphic to ${ }^{t} G r a(W)$ by the bijection on edges that exchanges $(2,1)$ and $(2,3)$, and $(2,2)$ and $(2,4)$.

If $W$ is not connected, ${ }^{t} \mathrm{Gra}(W)$ is defined as above but may not be connected. It is a $(2,2)$-regular graph with transitions. (It may still be connected if $W$ has empty components).

Lemma 24: A double occurrence multiword $W$ is is prime if and only if ${ }^{t} \mathrm{Gra}(W)$ is atomic and not empty.

Proof: Let $W=\left(w_{1}, w_{2}, \ldots, w_{n}\right) \in D O_{n}(V)$. We assume that it is not prime because of a bipartition $\left(V_{1}, V_{2}\right)$. Without loss of generality, we assume that $w_{1}=u v$ with $u \in V_{1}^{+}, v \in V_{2}^{+}, w_{2}, \ldots, w_{n} \in V_{1}^{+} \cup V_{2}^{+}$. We let $a$ be the 
first letter of $u, b$ be its last letter, $c$ be the first letter of $v$ and $d$ be its last letter. We may have $a=b, c=d$ and even $u=a=b$ or $v=c=d$. (An example is $(a c, a g g, c h h)$ that is not a Gauss multiword). The only edges of ${ }^{t} \operatorname{Gra}(W)$ between $V_{1}$ and $V_{2}$ are $e: b \rightarrow c$ and $f: d \rightarrow a$. It follows that $\operatorname{Graph}\left({ }^{t} \operatorname{Gra}(W)\right)=H \boxplus_{e, f} K$ for some graphs $H$ with vertex set $V_{1}$ and $K$ with vertex set $V_{2}$. Hence, ${ }^{t} \operatorname{Gra}(W)$ is not prime.

For the converse, we assume that $G=\operatorname{Graph}\left({ }^{t} \operatorname{Gra}(W)\right)=H \boxplus_{e, f} K$ for some graphs $H$ and $K$. Every closed walk of $G$ that goes through $e$ must go through $f$. The $t$-graph ${ }^{t} G r a(W)$ is covered by a union of pairwise edge-disjoint closed straight walk. One of them contains $e$ and $f$ and can be written $\operatorname{PPfQ}$ where $P$ and $Q$ are paths, respectively in $K$ and in $H$. The other closed straight walks are either in $H$ or in $K$. It follows that the bipartition $\left(V_{H}, V_{K}\right)$ of $V_{G}$ shows that $W$ is not prime.

\section{Proposition 25:}

(1) If $\left(\mathcal{C}_{1}, \ldots, \mathcal{C}_{n}\right)$ is a connected $n$-tuple of curves, then ${ }^{t} \operatorname{Gra}\left(W\left(\mathcal{C}_{1}, \ldots, \mathcal{C}_{n}\right)\right)$ is v-isomorphic to $G\left(\mathcal{C}_{1}, \ldots, \mathcal{C}_{n}\right)$, in such a way that the $i$-th closed straight walk of ${ }^{t} \operatorname{Gra}\left(W\left(\mathcal{C}_{1}, \ldots, \mathcal{C}_{n}\right)\right)$ is transformed into the $i$-th closed straight walk of $G\left(\mathcal{C}_{1}, \ldots\right.$, $\left.\mathcal{C}_{n}\right)$.

(2) Let $W \in D O_{n}(V)$ be connected. It is a Gauss multiword if and only if the $t$-graph ${ }^{t} \mathrm{Gra}(W)$ is planar.

Proof: $(1)$ We let $W\left(\mathcal{C}_{1}, \ldots, \mathcal{C}_{n}\right)=\left(w_{1}, \ldots, w_{n}\right)$, with each sequence $w_{i}$ nonempty and written $\left(v_{i, 1}, \ldots, v_{i, n_{i}}\right)$.We first assume that each segment $s_{i, j}$ of $\mathcal{C}_{i}$ links $v_{i, j}$ to $v_{i, j+1}$ (and $s_{i, n_{i}}$ links $v_{i, n_{i}}$ to $\left.v_{i, 1}\right)$. Then, ${ }^{t} \operatorname{Gra}\left(W\left(\mathcal{C}_{1}, \ldots, \mathcal{C}_{n}\right)\right)=$ $G\left(\mathcal{C}_{1}, \ldots, \mathcal{C}_{n}\right)$. Otherwise, a segment $s_{i, j}$ of $\mathcal{C}_{i}$ may link $v_{i, j^{\prime}}$ to $v_{i, j^{\prime}+1}$ and then, ${ }^{t} \operatorname{Gra}\left(W\left(\mathcal{C}_{1}, \ldots, \mathcal{C}_{n}\right)\right)$ and $G\left(\mathcal{C}_{1}, \ldots, \mathcal{C}_{n}\right)$ are v-isomorphic.

(2) Consider a plane embedding of the $t$-graph ${ }^{t} \operatorname{Gra}(W)$. Its closed straight walks define curves $\mathcal{C}_{1}, \ldots, \mathcal{C}_{n}$ such that $W=W\left(\mathcal{C}_{1}, \ldots, \mathcal{C}_{n}\right)$. Conversely, if $W$ is a Gauss multiword defined as $W\left(\mathcal{C}_{1}, \ldots, \mathcal{C}_{n}\right)$, then $\mathcal{C}_{1}, \ldots, \mathcal{C}_{n}$ form a plane embedding of ${ }^{t} G r a(W)$

The second assertion of this proposition yields a natural characterization of the Gauss multiwords and a linear-time recognition algorithm based on that of Proposition 15.

Corollary 26: Let $W \in D O_{n}(V)$ be a connected Gauss multiword.

(1) The tuples of curves $\left(\mathcal{C}_{1}, \ldots, \mathcal{C}_{n}\right)$ such that $W\left(\mathcal{C}_{1}, \ldots, \mathcal{C}_{n}\right)=W$ are obtained from the plane embeddings $\mathcal{E}$ of the $t$-graph ${ }^{t} \operatorname{Gra}(W)$ by defining $\mathcal{C}_{i}=\mathcal{E}\left(\omega_{i}\right)$ where $\omega_{i}$ is the closed straight walk of ${ }^{t} G r a(W)$ consisting of the edges $(i, j)$.

(2) Two such tuples are homeomorphic if and only the corresponding embeddings of ${ }^{t} \mathrm{Gra}(W)$ are homeomorphic.

Proof: (1) Clear from the previous constructions.

(2) If $\mathcal{E}$ and $\mathcal{E}^{\prime}$ are two homeomorphic embeddings of ${ }^{t} \mathrm{Gra}(W)$, the corresponding tuples of curves are homeomorphic. 
The converse may look evident, but a careful proof is necessary. Assume that $\varphi: \mathbb{S} \rightarrow \mathbb{S}$ is a homeomorphism of the sphere $\mathbb{S}$ to itself that maps $\left(\mathcal{C}_{1}, \ldots, \mathcal{C}_{n}\right)$ defined from $\mathcal{E}$ to $\left(\mathcal{C}_{1}^{\prime}, \ldots, \mathcal{C}_{n}^{\prime}\right)$ defined from $\mathcal{E}^{\prime}$. Since the intersection points are designated by the elements of $V$, we have $\varphi(\mathcal{E}(v))=\mathcal{E}^{\prime}(v)$ for each $v \in V$. Furthermore, $\varphi\left(\mathcal{E}\left(\omega_{i}\right)\right)=\mathcal{E}^{\prime}\left(\omega_{i}\right)$ for each $i$. Hence, for each edge $(i, j)$ we have: $\varphi(\mathcal{E}((i, j)))=\mathcal{E}^{\prime}\left(\left(i, j^{\prime}\right)\right)$ where $j^{\prime}$ may be different from $j$.

Let us examine this case: the edges $(i, j)$ and $\left(i, j^{\prime}\right)$ have same tail and head. Since $\mathcal{E}$ and $\mathcal{E}^{\prime}$ preserve the transitions of ${ }^{t} G r a(W)$ and $\varphi$ maps $\mathcal{E}\left(\omega_{i}\right)$ to $\mathcal{E}^{\prime}\left(\omega_{i}\right)$, we also have $\varphi(\mathcal{E}((i, j+1)))=\mathcal{E}^{\prime}\left(\left(i, j^{\prime}+1\right)\right)$ and thus $(i, j+1)$ and $\left(i, j^{\prime}+1\right)$ have also same tail and head. Since every $v \in V$ has at most two occurrences in each walk $\omega_{i}$, this means that the tails of $(i, j)$ and $\left(i, j^{\prime}\right)$ are the two occurrences (in $W$ ) of some $v$, and the same is true for their heads, and for those of $(i, j+1)$ and $\left(i, j^{\prime}+1\right)$. Hence, since $W$ is connected, we have $n=1$ and $W=(w w)$ for some sequence $w$, hence is a square.

Hence, if $W$ is not a square, $\varphi(\mathcal{E}((i, j)))=\mathcal{E}^{\prime}\left(\left(i, j^{\prime}\right)\right)$ implies $j^{\prime}=j$, and $\mathcal{E}$ and $\mathcal{E}^{\prime}$ are homeomorphic embeddings of ${ }^{t} G r a(W)$. If $W$ is a square, then ${ }^{t} \mathrm{Gra}(W)$ is a planar $t$-atom (cf. Figure 2) and has a unique plane embedding up to homeomorphism by Theorem 16 . So the result holds in both cases.

The proof of Assertion (2) shows that, unless $W$ is a square, there is a unique v-automorphism of ${ }^{t} \mathrm{Gra}(W)$ that preserves each closed straight walk. If $W$ is a square there are two such v-automorphisms. Note for comparison that if $W=(w, w)$, then there is a v-automorphism of ${ }^{t} G r a(W)$ that exchanges the two closed straight walks.

\subsubsection{Unambiguous Gauss multiwords}

We say that a Gauss multiword $W$ is unambiguous if any two systems of curves that define it are homeomorphic. It is thus unambiguous if and only if ${ }^{t} G r a(W)$ has a unique embedding (by Corollary 26), hence if and only if it is an atom by Corollary 21. We now characterize this property in terms of $W$.

Theorem 27 : A connected Gauss multiword $W$ is unambiguous if and only if it is prime. If it is ambiguous, all tuples $\left(\mathcal{C}_{1}, \ldots, \mathcal{C}_{n}\right)$ such that $W\left(\mathcal{C}_{1}, \ldots, \mathcal{C}_{n}\right)=$ $W$ can be determined from the planar maps of the $t$-graph ${ }^{t} G r a(W)$ by means of any term over $t$-atoms that defines it.

Proof: A connected Gauss multiword $W$ that is unambigous if and only if ${ }^{t} \mathrm{Gra}(W)$ is atomic by Corollary 21 and ${ }^{t} \mathrm{Gra}(W)$ is atomic if and only if $W$ is prime by Lemma 24 .

If a connected Gauss multiword $W$ is ambiguous, the tuples of curves $\left(\mathcal{C}_{1}, \ldots, \mathcal{C}_{n}\right)$ such that $W\left(\mathcal{C}_{1}, \ldots, \mathcal{C}_{n}\right)=W$ are in bijection with the planar maps of the $t$ graph ${ }^{t} G r a(W)$ by Corollary 26. Theorem 20 and Corollary 21 show that these maps can be determined from any term over $t$-atoms that defines this $t$-graph. $\square$ 
If in a Gauss multiword $W=W\left(\mathcal{C}_{1}, \ldots, \mathcal{C}_{n}\right)$ defined by $\left(w_{1}, \ldots, w_{n}\right) \in\left(V^{*}\right)^{n}$ we replace some $w_{i}$ by $\widetilde{w_{i}}$, then we obtain the Gauss multiword $W^{\prime}$ corresponding to the same tuple of curves where the orientation of $\mathcal{C}_{i}$ is reversed. Hence, the ambiguity of $W$ does not depend on the orientations of the curves it represents. Furthermore, there is a bijective correspondence between the tuples of curves corresponding to $W$ and to $W^{\prime}$.

\subsubsection{Small components of multiwords.}

We now consider tuples of curves such that at least one curve has exactly two intersection points with the others. The associated multiwords have several components and at least one of them has length 2. By "reducing" these multiwords, we will be able to extend Theorem 27 to tuples of nonoriented curves.

Definition 28 : Small components and reduction.

(1) Let $W=\left(w_{1}, \ldots, w_{n}\right)$ be a Gauss multiword. A component $w_{i}$ of $W$ of the form $a b$ with $a \neq b$ is a small component. Some other component $w_{j}$ must contain $a$ and $b$. We define $W^{\prime}$ from $W$ by removing $w_{i}$ and the letters $a$ and $b$ from $w_{j}$; it is a Gauss multiword because, if $W$ is associated with a tuple of curves $\left(\mathcal{C}_{1}, \ldots, \mathcal{C}_{n}\right)$, then $W^{\prime}$ is associated with the $(n-1)$-tuple obtained from $\left(\mathcal{C}_{1}, \ldots, \mathcal{C}_{n}\right)$ by removing $\mathcal{C}_{i}$. We write this $W \rightarrow W^{\prime}$ and we call this transformation a reduction step. It is clear that $W$ is connected if and only if $W^{\prime}$ is. (Since $(\varepsilon)$ is defined as connected, this is true for $W=(a b, a b))$.

(2) A Gauss multiword $W$ is reducible if it has small components; if furthermore it is connected, either it is of the form $(a b, a b)$ or it can be written without loss of generality (by reordering its components if necessary):

$$
W=\left(a_{1} b_{1}, a_{2} b_{2}, \ldots, a_{p} b_{p}, w_{1}, \ldots, w_{m}\right)
$$

where $a_{1}, b_{1}, \ldots, a_{p}, b_{p}$, are pairwise distinct and each component $w_{1}, \ldots, w_{m}$ has length at least 4 . Each pair $a_{i}, b_{i}$ occurs in one of the components $w_{1}, \ldots, w_{m}$. We let $X=\left\{a_{1}, b_{1}, a_{2}, b_{2}, \ldots, a_{p}, b_{p}\right\}$ and we define $\operatorname{Red}(W)$ as $\left(w_{1}^{\prime}, \ldots, w_{m}^{\prime}\right)$ where $w_{i}^{\prime}:=w_{i} \uparrow(V-X)$ (cf. Section 5.1 .1 for notation). Clearly, $W \rightarrow^{*}$ $\operatorname{Red}(W)$. However, $\operatorname{Red}(W)$ may have small components that we do not remove. For an example $\operatorname{Red}((a b, c d, a b e f, c d e f))=(e f, e f)$.

Reduction viewed in terms of graphs.

Our objective is to show that if $W$ is a connected Gauss multiword that reduces into $W^{\prime}$ by the removal of a small component $a b$, then there exists a planar $t$-atom $D$ with vertices $a$ and $b$ such that (up to technical details):

$$
{ }^{t} G r a\left(W^{\prime}\right)=H \boxplus K \text { and } \quad{ }^{t} G r a(W)=H \boxplus D \boxplus K
$$


for some unique $t$-graphs $H$ and $K$ that can be defined from $W$. ( $H$ and/or $K$ may be empty).

Before starting the description of $H$ and $K$, we define $\mathbf{D}_{4}(x, y ; e, f, g, h)$ as the planar $t$-atom with vertices $x$ and $y$, edges $e, h: x \rightarrow y$ and $f, g: y \rightarrow x$ and transitions $(e, g),(g, e),(f, h)$ and $(h, f)$ (that yield the two closed straight walks $(e, g)$ and $(f, h))$. Figure 11 (right part) shows $\mathbf{D}_{4}\left(a, b ; g, f^{\prime}, g^{\prime}, e^{\prime}\right)$.

Let $W \in D O_{n}(V)$ be a connected Gauss multiword defined as $W\left(\mathcal{C}_{1}, \ldots, \mathcal{C}_{n}\right)$ where $\mathcal{C}_{1}$ has two intersections, $a$ and $b$, with one of the other curves. Without loss of generality (by reordering the tuple and taking a conjugate of $w_{2}$ ), we can assume that $W=\left(a b, a w_{2}^{\prime} b w_{2}^{\prime \prime}, w_{3}, \ldots, w_{n}\right)$. We have $W \rightarrow W^{\prime}=\left(w_{2}^{\prime} w_{2}^{\prime \prime}, w_{3}, \ldots, w_{n}\right)$.

We first assume that $w_{2}^{\prime}$ and $w_{2}^{\prime \prime}$ are not empty. The curve $\mathcal{C}_{1}$ separates the sphere into two connected open sets $F_{1}$ and $F_{2}$. (Our plane embeddings are in the sphere). Let $F_{1}$ be the one that contains the intersections represented by the letters of $w_{2}^{\prime}$. Let $V_{1}$ and $V_{2}$ be the set of letters of the components $w_{i}, 3 \leq i \leq n$, that are connected, respectively, to $w_{2}^{\prime}$ and to $w_{2}^{\prime \prime}$ via the components of $W$. The curves whose words are connected to $w_{2}^{\prime}$ (resp. to $w_{2}^{\prime \prime}$ ) are in $F_{1}$ (resp. in $F_{2}$ ), hence, $\left(V_{1}, V_{2}\right)$ is a bipartition of $V-\{a, b\}$. (If $W$ is not a Gauss multiword, then $V_{1}$ and $V_{2}$ may not be disjoint : take for instance $\left.W=(a b, a c b d, c d e e)\right)$.

We can reorder the components of $W$ so that $w_{2}^{\prime}, w_{3}, \ldots, w_{p} \in V_{1}^{*}$ and $w_{2}^{\prime \prime}, w_{p+1}, \ldots, w_{n} \in V_{2}^{*}$. Note that $W_{1}=\left(w_{2}^{\prime}, w_{3}, \ldots, w_{p}\right)$ and $W_{2}=\left(w_{2}^{\prime \prime}, w_{p+1}, \ldots, w_{n}\right)$ are connected Gauss multiwords, respectively $W\left(\mathcal{C}_{2}, \mathcal{C}_{3}, \ldots, \mathcal{C}_{p}\right)$ and $W\left(\mathcal{C}_{2}, \mathcal{C}_{p+1}, \ldots, \mathcal{C}_{n}\right)$.

We let $G:={ }^{t} \operatorname{Gra}(W), G^{\prime}:={ }^{t} G r a\left(W^{\prime}\right)$ and $G_{i}:={ }^{t} G r a\left(W_{i}\right)$ for $i=1,2$. We will denote in the same way the associated underlying graphs. In order to relate precisely $G_{1}$ and $G_{2}$ to $G$, we let $c$ and $c^{\prime}$ be respectively the first and last letter of $w_{2}^{\prime}$, and $d$ and $d^{\prime}$ be similarly the first and last element of $w_{2}^{\prime \prime}$. In $G$, we have the following edges that we name $e, e^{\prime}, f, f^{\prime}, g$ and $g^{\prime}$ :

$$
e: c^{\prime} \rightarrow b, e^{\prime}: a \rightarrow c, f: d^{\prime} \rightarrow a, f^{\prime}: b \rightarrow d, g: a \rightarrow b \text { and } g^{\prime}: b \rightarrow a
$$

cf. the left part of Figure 11.

Then $G_{1}$ is $G\left[V_{1}\right]$ augmented with an edge : $c^{\prime} \rightarrow c$ that we name $e$, and, similarly, $G_{2}$ is $G\left[V_{2}\right]$ augmented with $f: d^{\prime} \rightarrow d$. The transitions making them into $t$-graphs are as follows: $\tau_{G_{1}}\left(e^{+}\right):=\tau_{G}\left(e^{\prime+}\right)$ and $\tau_{G_{2}}\left(f^{+}\right):=\tau_{G}\left(f^{\prime+}\right)$; otherwise, $\tau_{G_{1}}(x)$ and $\tau_{G_{2}}(x)$ are $\tau_{G}(x)$. It is then clear that

$$
G^{\prime}=G_{1} \boxplus_{e, f} G_{2} \text {.and } G=G_{1} \boxplus_{e, e^{\prime}} \mathbf{D}_{4}\left(a, b ; g, f^{\prime}, g^{\prime}, e^{\prime}\right) \boxplus_{f^{\prime}, f} G_{2} .
$$

If $w_{2}^{\prime} \neq \varepsilon$ and $w_{2}^{\prime \prime}=\varepsilon$, then $V_{1} \neq \emptyset, V_{2}=\emptyset$ and $G_{2}$ is undefined. In $G:={ }^{t} \operatorname{Gra}(W)$, we have the following edges:

$e: c^{\prime} \rightarrow b, e^{\prime}: a \rightarrow c, f^{\prime}: b \rightarrow a, g: a \rightarrow b$ and $g^{\prime}: b \rightarrow a$

with transitions $\left(e, f^{\prime}\right),\left(f^{\prime}, e^{\prime}\right),\left(g, g^{\prime}\right)$ and $\left(g^{\prime}, g\right)$.

Here, $W^{\prime}=\left(w_{2}^{\prime}, w_{3}, \ldots, w_{n}\right)$. and we have :

$$
G:={ }^{t} G r a\left(W^{\prime}\right) \boxplus_{e, e^{\prime}} \mathbf{D}_{4}\left(a, b ; g, f^{\prime}, g^{\prime}, e^{\prime}\right) .
$$



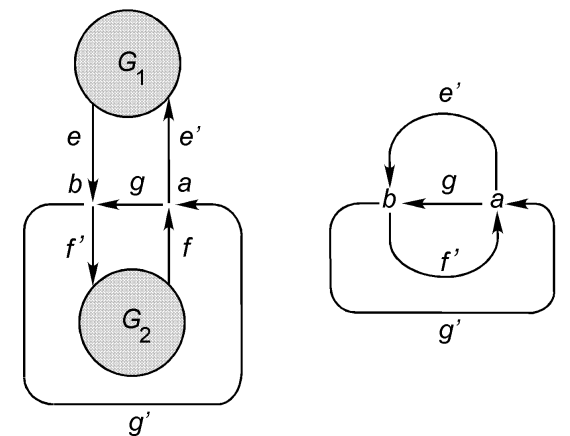

Figure 11: Assertion (1) of Proposition 29

If $w_{2}^{\prime}=w_{2}^{\prime \prime}=\varepsilon$, then $V_{1}=V_{2}=\emptyset, W=(a b, a b), W^{\prime}=(\varepsilon)$ and:

${ }^{t} G r a(W)=\mathbf{D}_{4}\left(a, b ; g, f^{\prime}, g^{\prime}, e^{\prime}\right)$

for an appropriate naming of the edges.

These constructions establish the following:

Proposition 29: Let $W=\left(a b, a w_{2}^{\prime} b w_{2}^{\prime \prime}, w_{3}, \ldots, w_{n}\right)$ be a connected Gauss multiword with a small component $a b$ that reduces to $W^{\prime}=\left(w_{2}^{\prime} w_{2}^{\prime \prime}, w_{3}, \ldots, w_{n}\right)$.

(1) If $w_{2}^{\prime}, w_{2}^{\prime \prime} \neq \varepsilon$, then ${ }^{t} G r a\left(W^{\prime}\right)=G_{1} \boxplus_{e, f} G_{2}$ and $G=G_{1} \boxplus_{e, e^{\prime}}$ $\mathbf{D}_{4}\left(a, b ; g, f^{\prime}, g^{\prime}, e^{\prime}\right) \boxplus_{f^{\prime}, f} G_{2}$,

(2) if $w_{2}^{\prime} \neq \varepsilon$ and $w_{2}^{\prime \prime}=\varepsilon$, then ${ }^{t} G r a\left(W^{\prime}\right)={ }^{t} G r a\left(W^{\prime}\right) \boxplus_{e, e^{\prime}} \mathbf{D}_{4}\left(a, b ; g, f^{\prime}, g^{\prime}, e^{\prime}\right)$,

(3) if $w_{2}^{\prime}=w_{2}^{\prime \prime}=\varepsilon$, then ${ }^{t} G r a(W)=\mathbf{D}_{4}\left(a, b ; g, f^{\prime}, g^{\prime}, e^{\prime}\right)$,

where $G_{1}, G_{2}, e^{\prime}, e^{\prime}$ etc. are as in the construction. $\square$

Remarks 30: (1) The order in which the edges occur in $\mathbf{D}_{4}\left(a, b ; g, f^{\prime}, g^{\prime}, e^{\prime}\right)$ in Proposition 29 is important. Figure 12 shows for comparison the graph

$$
H=G_{1} \boxplus_{e, e^{\prime}} \mathbf{D}_{4}\left(a, b ; g, g^{\prime}, f^{\prime}, e^{\prime}\right) \boxplus_{f^{\prime}, f} G_{2} .
$$

The corresponding Gauss multiword is $W=\left(b a w_{2}^{\prime}, a b w_{2}^{\prime \prime}, w_{3}, \ldots, w_{n}\right)$.

(2) Proposition 29 and the unicity assertion of Theorem 14 show that ${ }^{t} G r a\left(W^{\prime}\right)$ has exactly one atom less than ${ }^{t} G r a(W)$ : this atom is $\mathbf{D}_{4}\left(a, b ; g, f^{\prime}, g^{\prime}, e^{\prime}\right)$.

Construction of curves.

A face of a plane embedding $\mathcal{E}$ of a graph $G$ is a connected component of $\mathbb{S}-\mathcal{E}$. It is an open set and its (topological) border is the union of some line segments representing edges of $G$. 


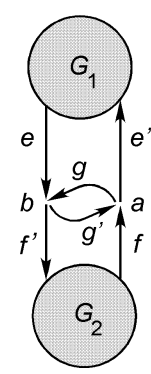

Figure 12: The graph $H$ of Remark 30(1).

Lemma 31: A plane embedding of a graph $G=H \boxplus_{e, f} K \in \mathcal{S C}$ has two faces whose borders contain the line segments representing $e$ and $f$.

Proof: Let $\mathcal{E}$ be a plane embedding of $G=H \boxplus_{e, f} K \in \mathcal{S C}$. The graphs $H-e$ and $K-f$ are connected, disjoint and $G-\{e, f\}=(H-e) \cup(K-f)$. The restriction of $\mathcal{E}$ to $G-\{e, f\}$, denoted by $\mathcal{E}\lceil(G-\{e, f\})$ is the union of $\mathcal{E}\left\lceil(H-e)\right.$ and $\mathcal{E}\left\lceil(K-f)\right.$. Then, $\mathcal{E} \uparrow(H-e)$ is included in a face $F_{K}$ of $\mathcal{E}\lceil(K-f)$ whose border we denote by $B$. Similarly, $\mathcal{E}\lceil(K-f)$ is included in a face $F_{H}$ of $\mathcal{E}\left\lceil(H-e)\right.$ whose border we denote by $B^{\prime}$. Then $\mathcal{E}\lceil(G-\{e, f\})$ has a face $F=F_{H} \cap F_{K}$ whose border is $B \cup B^{\prime}$. Since $\mathcal{E}(e) \cap F \neq \emptyset$ and $\mathcal{E}(f)$ $\cap F \neq \emptyset$, each of $e$ and $f$ has one end vertex in $B$ and the other in $B^{\prime}$ (otherwise $\mathcal{E}$ is not plane). It follows that $\mathcal{E}(e)$ and $\mathcal{E}(f)$ divide $F$ into two faces of $\mathcal{E}$ whose borders contain both of them.

Proposition 32 : Let $W$ be a connected Gauss multiword that reduces to $W^{\prime}$ by deletion of one small component, and $\mathcal{S}^{\prime}$ be a tuple of oriented curves such that $W^{\prime}=W\left(\mathcal{S}^{\prime}\right)$.

(1) If $W^{\prime}=(\varepsilon)$, there is a unique way, up to homeomorphism, to extend $\mathcal{S}^{\prime}$ into a pair $\mathcal{S}$ such that $W(\mathcal{S})=W$.

(2) If $W^{\prime} \neq(\varepsilon)$, there are exactly two ways, up to homeomorphism, to extend $\mathcal{S}^{\prime}$ into a tuple $\mathcal{S}$ such that $W(\mathcal{S})=W$.

Proof: (1) In this case, $W=(a b, a b), \mathcal{S}^{\prime}$ is one circle (without self-intersection), and there is a unique way to extend $\mathcal{S}^{\prime}$ into $\mathcal{S}$ such that $W(\mathcal{S})=(a b, a b)$ because ${ }^{t} G r a(W)$ is a $t$-atom.

(2) Assume first that ${ }^{t} G r a\left(W^{\prime}\right)=G_{1} \boxplus_{e, f} G_{2}$ (cf. Proposition 29(1)).and consider its embedding $\mathcal{E}^{\prime}$ defined by $\mathcal{S}^{\prime}$. One of its curves contains $\mathcal{E}^{\prime}(e)$ and $\mathcal{E}^{\prime}(f)$. Note that the edges $e$ and $f$ are the unique ones in ${ }^{t} G r a\left(W^{\prime}\right)$ that link respectively $V_{1}$ to $V_{2}$ (i.e., a vertex of $V_{1}$ to a vertex of $V_{2}$ ) and $V_{2}$ to $V_{1}$ (so that $\mathcal{E}^{\prime}(e)$ and $\mathcal{E}^{\prime}(f)$ are defined in a unique way as segments of a line). The curve to be added to $\mathcal{S}^{\prime}$ must cross these two segments and no other one. By Lemma $31, \mathcal{E}^{\prime}$ has two faces $F$ and $F^{\prime}$ whose borders contain these segments. Let us subdivide these segments by adding two points $x_{e}$ and $x_{f}$ representing 
the two vertices of ${ }^{t} G r a(W)$ not in ${ }^{t} G r a\left(W^{\prime}\right)$. The $t$-graph ${ }^{t} G r a(W)$ has edges $g: x_{f} \rightarrow x_{e}$ and $g^{\prime}: x_{e} \rightarrow x_{f}$ with transitions $\left(g, g^{\prime}\right)$ and $\left(g^{\prime}, g\right)$. We make $\mathcal{E}^{\prime}$ into a plane embedding $\mathcal{E}$ of ${ }^{t} \mathrm{Gra}(W)$ by adding line segments in exactly two possible ways (up to homeomorphism): we can draw $g$ in $F$ and $g^{\prime}$ in $F^{\prime}$ or vice versa. Figure 11 shows one possibilty. The other one is obtained by exchanging $g$ and $g^{\prime}$.

If now that $V_{2}=\emptyset$ then $G={ }^{t} G r a\left(W^{\prime}\right) \boxplus_{e, e^{\prime}} \mathbf{D}_{4}\left(a, b ; g, f^{\prime}, g^{\prime}, e^{\prime}\right)$ (Proposition $29(2))$. Consider the embedding $\mathcal{E}^{\prime}$ of ${ }^{t} G r a\left(W^{\prime}\right)$ defined by $\mathcal{S}^{\prime}$. The edge $e$ of ${ }^{t} G r a\left(W^{\prime}\right)$ links $c$ to $c^{\prime}$ that are respectively the first and last element of $w_{2}^{\prime}$, cf. Lemma 31. If $W^{\prime}$ is not a square, there is a unique segment of some line that is $\mathcal{E}^{\prime}(e)$, see Corollary 26(2). The new line must intersect it twice, at $a$ and $b$, in the order $c, b, a, c^{\prime}$. Again there are exactly two ways, up to homeomorphism to define it. If $W^{\prime}$ is a square $(w w)$, there are two line segments representing edges from $c$ to $c^{\prime}$. This seems to yield four ways to extend $\mathcal{S}^{\prime}$ into $\mathcal{S}$, but actually we get only two, up to homeomorphism, because these two segments correspond to two edges related by a v-automorphism of ${ }^{t} G r a\left(W^{\prime}\right)$.

Anticipating on the sequel, we observe that in Case (2), there are two ways to extend $\mathcal{S}^{\prime}$ into $\mathcal{S}$ because the curves are oriented. If we consider nonoriented curves, we get a unique extension, up to homeomorphism.

\subsection{Curves without orientation}

We now consider curves $\mathcal{C}_{1}, \ldots, \mathcal{C}_{n}$ having no particular orientation. The components of $W\left(\mathcal{C}_{1}, \ldots, \mathcal{C}_{n}\right)$ are thus reversal-circular: we need not distinguish a sequence from its reversal. For the three nonoriented curves of Figure 3, we get $W\left(\mathcal{C}_{1}, \mathcal{C}_{2}, \mathcal{C}_{3}\right)$ defined by $(a b c d, a e c f, b f d e)$ as well as by $(a b c d, f c e a, b f d e)$ or $(c d a b, a f c e, \operatorname{debf})$.

Consider again the oriented curves of Figure 4. The corresponding multiword $W=(a b c d, b c, a d)$ is ambiguous as already observed. However, if we omit the orientations, the two triples of curves are homeomorphic and $W$ is not ambiguous for describing nonoriented curves. The difference between the oriented and nonoriented cases is due to the presence of curves with two intersections because reversing the orientation of such a curve that has two crossings with the others changes the corresponding map in $\mathcal{P} \mathcal{M}_{2,2}^{t}$ but not its undirected version.

If the curves of a tuple $\left(\mathcal{C}_{1}, \ldots, \mathcal{C}_{n}\right)$ are not oriented, we define $G\left(\mathcal{C}_{1}, \ldots, \mathcal{C}_{n}\right)$ as $\operatorname{Und}\left(G\left(\mathcal{C}_{1}^{\prime}, \ldots, \mathcal{C}_{n}^{\prime}\right)\right)$ where $\mathcal{C}_{i}^{\prime}$ is an orientation of $\mathcal{C}_{i}$.

Let $\left(\mathcal{C}_{1}, \ldots, \mathcal{C}_{n}\right)$ and $\left(\mathcal{C}_{1}^{\prime}, \ldots, \mathcal{C}_{n}^{\prime}\right)$ be two tuples of oriented curves such that $W\left(\mathcal{C}_{1}, \ldots, \mathcal{C}_{n}\right) \asymp W\left(\mathcal{C}_{1}^{\prime}, \ldots, \mathcal{C}_{n}^{\prime}\right)$. (The equivalence $\asymp$ combines conjugacy and reversal.) If they are homeomorphic, then, the tuples of corresponding nonoriented curves are also homeomorphic. Hence if $W\left(\mathcal{C}_{1}, \ldots, \mathcal{C}_{n}\right)=W$ is ambiguous 
for nonoriented curves, it is also for oriented ones. The converse is not always true. However, it is for a multiword $W$ provided the nonoriented curves of any tuple that yields it can be equipped with an orientation depending only on $W$. This fact motivates the following definition.

Definition 33 : Orientable components of multiwords.

(1) A reversal-circular sequence in $V^{\odot}$ is orientable if it is defined by $w \in V^{*}$ such that $\operatorname{Conj}(w) \neq \operatorname{Conj}(\widetilde{w})(\operatorname{Conj}(w)$ is the set of conjugate words of $w$, cf. Section 5.1.1). It is easy to see that, if this condition is true for $w$, then it is also true for every sequence $\asymp$-equivalent to $w$. A palindrome is a sequence $w$ such that $w=\widetilde{w}$. A sequence $\asymp$-equivalent to a palindrome is not orientable. Neither is $a b$, where $a, b \in V$.

(2) Let $\leq$ be an arbitrary but fixed linear order on $V$. From it we get a lexicographic linear order on the set of finite subsets of $V^{*}$, that we also denote by $\leq$. For every orientable $w \in V^{*}$, we define:

(2.1) $\mu(w)=w$ if $\operatorname{Conj}(w)<\operatorname{Conj}(\widetilde{w})$, and

$(2.2) \mu(w)=\widetilde{w}$ if $\operatorname{Conj}(\widetilde{w})<\operatorname{Conj}(w)$.

If all components of $W=\left(w_{1}, \ldots, w_{n}\right) \in\left(V^{*}\right)^{n}$ are orientable, we define:

$$
\mu(W)=\left(\mu\left(w_{1}\right), \ldots, \mu\left(w_{n}\right)\right) .
$$

Lemma 34 : A component of a Gauss multiword is orientable except if it is (conjugate to) a palindrome or has length 2 .

Proof: In the following proof, $a, b, c$ denote elements of $V$ and $u, v, w, w_{1}$ etc. denote elements of $V^{*}$.

Fact: A Gauss multiword has no component of the form aub $\widetilde{u}$ or aua $\widetilde{u}$ with $u \neq \varepsilon$. (Otherwise, by constructing the closed straight walk associated with $a u b \widetilde{u}$ or $a u a \widetilde{u}$ we get a contradiction with planarity)

Let $w$ be a component of a Gauss multiword such that $\operatorname{Conj}(w)=\operatorname{Conj}(\widetilde{w})$. We prove that it is of the form $a b$, with $a \neq b$ or is conjugate to a palindrome. We distinguish several cases. Recall that $w$ can be replaced by any conjugate sequence, and that its length is even.

Case 1: $w$ has at least two different letters $a, b$ that have only one occurrence. Then $w=a u b v$ and $w \in \operatorname{Conj}(\widetilde{w})=\operatorname{Conj}(\widetilde{v} b \widetilde{u} a)$. We must have $w=a \widetilde{v} b \widetilde{u}$, hence $v=\widetilde{u}$. But this contradicts the initially observed fact, except if $u=v=\varepsilon$, which gives $w=a b$.

In the next two cases, all letters in $w$ have two occurrences in this word.

Case 2 : $w=u v$ where $u$ and $v$ are sequences over two disjoint nonempty alphabets (or $w$ is conjugate to such a sequence). Then $w \in \operatorname{Conj}(\widetilde{w})=\operatorname{Conj}(\widetilde{v} \widetilde{u})$ 
implies $w=\widetilde{u} \widetilde{v}$, hence $u=\widetilde{u}$ and $v=\widetilde{v}$ are two palindromes. If they have both even length, then $w=u_{1} \widetilde{u_{1}} v_{1} \widetilde{v_{1}}$. It is conjugate to the palindrome $\widetilde{u_{1}} v_{1} \widetilde{v_{1}} u_{1}$. If they have odd length, then $w=u_{1} a \widetilde{u_{1}} v_{1} b \widetilde{v_{1}}$. But $a$ and $b$ belong to disjoint alphabets, hence, they cannot have two occurrences. This case cannot happen.

The first letter of $w$ differs from the last one. The only remaining possibilities are:

Case 3: $w=a u a v b x b$ and $w=a u b v a x b$.

In the first case, we have $w \in \operatorname{Conj}(b \widetilde{x} b \widetilde{v} a \widetilde{u} a)$. We must have auavbxb= $a \widetilde{u} a b \widetilde{x} b \widetilde{v}$, hence, $v=\varepsilon$ and $u$ and $x$ are palindromes. If $u$ and $x$ have even length, then $w$ is conjugate to a palindrome (cf. Case 2). If they have odd length, we have $w=a u_{1} c \widetilde{u_{1}} a b x_{1} c \widetilde{x_{1}} b$, hence $w$ is conjugate to $c \widetilde{u_{1}} a b x_{1} c \widetilde{x_{1}} b a u_{1}$ which contradicts the initial fact.

Very similar arguments using the initial fact eliminate the case where $w=$ aubvaxb.

If $\left(\mathcal{C}_{1}, \ldots, \mathcal{C}_{n}\right)$ is an $n$-tuple of oriented curves, we denote by $N O\left(\mathcal{C}_{1}, \ldots, \mathcal{C}_{n}\right)$ the tuple of nonoriented curves obtained by forgetting the orientations.

Proposition 35 : Let $W$ be a connected Gauss multiword that is orientable or has a single component.

(1) $W$ is unambiguous if and only if it is for describing nonoriented curves.

(2) If $W$ is ambiguous, there is a bijection that preserves homeomorphisms between the $n$-tuples of oriented and of nonoriented curves described by $W$. Both sets of $n$-tuples can be described from any term over atoms that defines ${ }^{t} \operatorname{Gra}(W)$.

Proof: We first assume that $W=\left(w_{1}, \ldots, w_{n}\right)$ is orientable.

Claim: There exists a bijection $\nu$ between the $n$-tuples $\left(\mathcal{C}_{1}, \ldots, \mathcal{C}_{n}\right)$ of nonoriented curves described by $W$ and the $n$-tuples of oriented ones described by $\mu(W)$ such that $\left(\mathcal{C}_{1}, \ldots, \mathcal{C}_{n}\right)=N O\left(\nu\left(\mathcal{C}_{1}, \ldots, \mathcal{C}_{n}\right)\right)$. Furthermore, if $\left(\mathcal{D}_{1}, \ldots, \mathcal{D}_{n}\right)$ is homeomorphic to $\left(\mathcal{C}_{1}, \ldots, \mathcal{C}_{n}\right)$, then $\nu\left(\mathcal{D}_{1}, \ldots, \mathcal{D}_{n}\right)$ is homeomorphic to $\nu\left(\mathcal{C}_{1}, \ldots, \mathcal{C}_{n}\right)$ by the same homeomorphism of the sphere to itself.

Proof of the claim: Let $W$ and $\left(\mathcal{C}_{1}, \ldots, \mathcal{C}_{n}\right)$ be as stated. Then $\left(\mathcal{C}_{1}, \ldots, \mathcal{C}_{n}\right)=$ $N O\left(\mathcal{C}_{1}^{\prime \prime}, \ldots, \mathcal{C}_{n}^{\prime \prime}\right)$ where $\mathcal{C}_{1}^{\prime \prime}, \ldots, \mathcal{C}_{n}^{\prime \prime}$ are oriented curves such that $W=W\left(\mathcal{C}_{1}^{\prime \prime}, \ldots, \mathcal{C}_{n}^{\prime \prime}\right)$. Let $I$ be the set of indices $i$ such that $\mu\left(w_{i}\right)=\widetilde{w_{i}}$ (i.e., Case (2.2) of Definition 33 applies). Then we define $\nu\left(\mathcal{C}_{1}, \ldots, \mathcal{C}_{n}\right)$ as the $n$-tuple $\left(\mathcal{C}_{1}^{\prime \prime}, \ldots, \mathcal{C}_{n}^{\prime \prime}\right)$ except that we reverse the orientation of $\mathcal{C}_{i}^{\prime \prime}$ whenever $i \in I$.

It is then clear that $\operatorname{NO}\left(\nu\left(\mathcal{C}_{1}, \ldots, \mathcal{C}_{n}\right)\right)=\left(\mathcal{C}_{1}, \ldots, \mathcal{C}_{n}\right)$. The mapping $\nu$ is a bijection because the orientations of the curves in $\nu\left(\mathcal{C}_{1}, \ldots, \mathcal{C}_{n}\right)$ are determined from $W$. The last assertion is also clear.

This claim and the remark after Theorem 27 show that there is a bijection between the homeomorphism classes of the $n$-tuples of nonoriented and of oriented curves described by $W$, which proves (1) and (2) for orientable multiwords. 
It remains to consider the case where $n=1$ and $W$ is not orientable. By Lemma 34, this means that $W=(w)$ and $w$ is a palindrome.

Let $M$ be a planar map of ${ }^{t} \operatorname{Gra}(W)$. The map $M^{\prime}$ obtained from $M$ by reversing all edge directions is a planar map of ${ }^{t} G r a((\widetilde{w}))$ that is v-isomorphic to $M^{-1}$. Hence, up to homeomorphism, the same oriented and nonoriented curves are described by $w$ and by $\widetilde{w}$ which proves (1) and (2). Note that ${ }^{t} G r a(W)$ is prime, hence that $w$ is unambiguous if and only if $w=a a$.

With the hypotheses of this proposition, if $p$ is the number of atoms of the $t$-graph ${ }^{t} G r a(W)$, then the number of pairwise nonhomeomorphic tuples of oriented (resp. nonoriented) curves described by $W$ is $2^{p-1}$ by Corollary 21 . Another consequence of this proposition is that a Gauss word (representing a single curve) is unambiguous for describing nonoriented curves if and only if it is prime. This fact is proved in a completely different way in [ChaWeb].

We now use the reduction of Definition 28 to handle the general case.

Lemma 36 : Let $W$ be a connected Gauss multiword $W$ that is not a palindrome. There is a bijection $\theta$ between the homeomorphism classes of the tuples of nonoriented curves described by $W$ and those of oriented curves described by $\operatorname{Red}(W)$.

Proof: Let $W$ be orientable and defined by $W=\left(a_{1} b_{1}, a_{2} b_{2}, \ldots, a_{p} b_{p}, w_{1}, \ldots\right.$, $\left.w_{m}\right) \in\left(V^{*}\right)^{n}$ as in Definition 28. The sequences $\mu\left(w_{1}\right), \ldots, \mu\left(w_{m}\right)$ are defined because $w_{1}, \ldots, w_{m}$ have length at least 4 and are not palindromes. We let $w_{i}^{\prime}:=\mu\left(w_{i}\right) \uparrow\left(V-\left\{a_{1}, \ldots, a_{p}, b_{1}, \ldots, b_{p}\right\}\right.$. Hence, $\operatorname{Red}(W) \asymp\left(w_{1}^{\prime}, \ldots, w_{m}^{\prime}\right)$.

For every $n$-tuple of nonoriented curves $\left(\mathcal{C}_{1}, \ldots, \mathcal{C}_{n}\right)$ such that $W\left(\mathcal{C}_{1}, \ldots, \mathcal{C}_{n}\right) \asymp$ $W$, we let $\left(\mathcal{C}_{1}^{\prime}, \ldots, \mathcal{C}_{n}^{\prime}\right)$ be an $n$-tuple of oriented curves such that $\operatorname{NO}\left(\mathcal{C}_{1}^{\prime}, \ldots, \mathcal{C}_{n}^{\prime}\right)=$ $\left(\mathcal{C}_{1}, \ldots, \mathcal{C}_{n}\right)$ and $w\left(\mathcal{C}_{i}^{\prime}\right)=\mu\left(w_{i-p}\right)$ for each $i=p+1, \ldots, n$. Hence, the curves $\mathcal{C}_{p+1}^{\prime}, \ldots, \mathcal{C}_{n}^{\prime}$ have a canonical orientation based on the orientability of their associated sequences. For the curves $\mathcal{C}_{1}^{\prime}, \ldots, \mathcal{C}_{p}^{\prime}$, we take any orientation. It is then clear that $W\left(\mathcal{C}_{p+1}^{\prime}, \ldots, \mathcal{C}_{n}^{\prime}\right)=\left(w_{1}^{\prime}, \ldots, w_{m}^{\prime}\right)$. We define $\theta\left(\mathcal{C}_{1}, \ldots, \mathcal{C}_{n}\right)$ as $\left(\mathcal{C}_{p+1}^{\prime}, \ldots, \mathcal{C}_{n}^{\prime}\right)$.

Every $m$-tuple of oriented curves $\left(\mathcal{E}_{1}, \ldots, \mathcal{E}_{m}\right)$ such that $W\left(\mathcal{E}_{1}, \ldots, \mathcal{E}_{m}\right)=$ $\left(w_{1}^{\prime}, \ldots, w_{m}^{\prime}\right)$ is $\theta\left(\mathcal{C}_{1}, \ldots, \mathcal{C}_{n}\right)$ for some $\mathcal{C}_{1}, \ldots, \mathcal{C}_{n}$ such that $W\left(\mathcal{C}_{1}, \ldots, \mathcal{C}_{n}\right) \asymp W$.

Let $\left(\mathcal{D}_{1}, \ldots, \mathcal{D}_{n}\right)$ be an $n$-tuple of nonoriented curves such that $W\left(\mathcal{D}_{1}, \ldots, \mathcal{D}_{n}\right) \asymp$ $W$. If it is homeomorphic to $\left(\mathcal{C}_{1}, \ldots, \mathcal{C}_{n}\right)$, then $\theta\left(\mathcal{D}_{1}, \ldots, \mathcal{D}_{n}\right)$ is homeomorphic to $\theta\left(\mathcal{C}_{1}, \ldots, \mathcal{C}_{n}\right)$. This is so because the $n$-tuple $\left(\mathcal{D}_{1}^{\prime}, \ldots, \mathcal{D}_{n}^{\prime}\right)$ associated with $\left(\mathcal{D}_{1}, \ldots, \mathcal{D}_{n}\right)$ as $\left(\mathcal{C}_{1}^{\prime}, \ldots, \mathcal{C}_{n}^{\prime}\right)$ is with $\left(\mathcal{C}_{1}, \ldots, \mathcal{C}_{n}\right)$ is homeomorphic to $\left(\mathcal{C}_{1}^{\prime}, \ldots, \mathcal{C}_{n}^{\prime}\right)$ up to the orientations of the curves $\mathcal{D}_{1}^{\prime}, \ldots, \mathcal{D}_{p}^{\prime}$ that have two intersections with the others.

Note that $\operatorname{NO}\left(\theta\left(\mathcal{C}_{1}, \ldots, \mathcal{C}_{n}\right)\right)=\left(\mathcal{C}_{p+1}, \ldots, \mathcal{C}_{n}\right)$. Proposition 32 shows that, up to homeomorphism, there is a unique way to extend this $(n-p)$-tuple by nonoriented curves $\mathcal{C}_{1}, \ldots, \mathcal{C}_{p}$ in such a way that $W\left(\mathcal{C}_{1}, \ldots, \mathcal{C}_{n}\right) \asymp W$. This observation shows that if $\theta\left(\mathcal{D}_{1}, \ldots, \mathcal{D}_{n}\right)$ is homeomorphic to $\theta\left(\mathcal{C}_{1}, \ldots, \mathcal{C}_{n}\right)$, then $\left(\mathcal{D}_{1}, \ldots, \mathcal{D}_{n}\right)$ is homeomorphic to $\left(\mathcal{C}_{1}, \ldots, \mathcal{C}_{n}\right)$. 


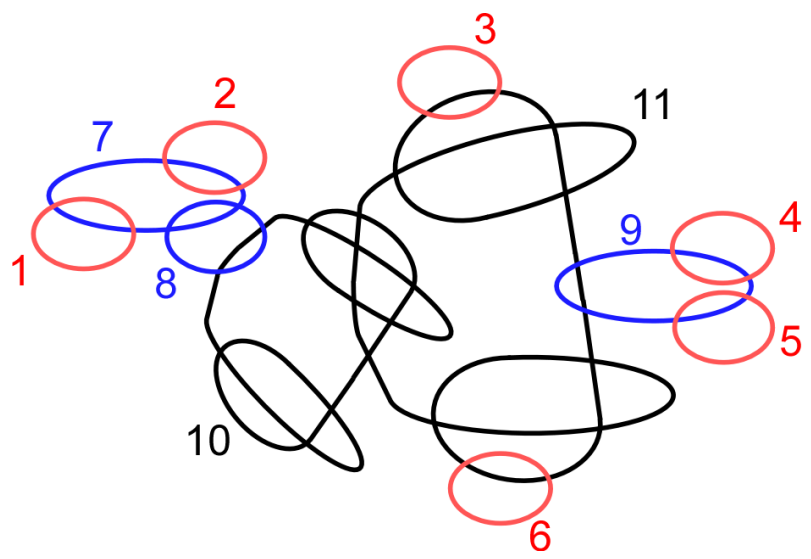

Figure 13: An 11-tuple of nonoriented curves.

Figure 13 shows an 11-tuple of nonoriented curves. The small components of the associated multiword $W$ correspond to curves 1 to 6 . The reduced multiword $\operatorname{Red}(W)$ has small components corresponding to curves 7 and 9.

\section{Algorithm 37 : Analysis of a double occurrence multiword.}

Let be given a double occurrence multiword $W$ intended to describe nonoriented curves.

1. We first check if it is connected. If it is not, we treat separately each of its connected submultiwords.

2. We construct the $t$-graph ${ }^{t} G r a(W)$ and we check its planarity.

3. If it is planar, then $W$ is a connected Gauss multiword, and we reduce it into $W^{\prime}=\operatorname{Red}(W)$ (cf. Definition 28) by keeping track of the reduction steps.

4. We construct the (planar) $t$-graph ${ }^{t} G r a\left(W^{\prime}\right)$ and a term $t$ over atoms that defines ${ }^{t} G r a\left(W^{\prime}\right)$ (we use Theorem 14). Let $p$ be the number of atoms used by $t$.

5. By using Proposition 15, we determine a planar $t$-map for each $t$-atom occurring in $t$.

6. By using Corollary 21, we compute the $2^{p-1}$ (terms defining the) planar $t$-maps that represent the different plane embeddings of ${ }^{t} \operatorname{Gra}\left(W^{\prime}\right)$, hence, the different tuples of curves that yield $W^{\prime}$.

7. Since we have recorded the sequence of reductions done at Step 3, and by using this sequence backwards, we can compute the $2^{p-1}$ planar $t$-maps of ${ }^{t} \operatorname{Gra}(W)$, hence, the different tuples of curves (extending those we have by step 6) described by $W$. 


\title{
6 Some open questions
}

\begin{abstract}
Knots and knot diagrams
The reader will find the definitions and basic facts in the books [Ada], [Bol] and [GodRoy]. Let us consider oriented links and knots. A (connected) link diagram is nothing but a map in $\mathcal{M}_{2,2}^{t}$ whose vertices are labelled by over or under, to indicate the type of crossing. A knot diagram is such a map that has a unique straight walk. We let $k$ map a diagram to the corresponding link or knot. Knots can be composed by a binary (multivalued) operation denoted in [Ada] by \# such that for every two knot diagrams $D$ and $D^{\prime}$, every edge $e$ of $D$ and every edge $f$ of $D^{\prime}$, we have $k\left(D \boxplus_{e, f} D^{\prime}\right)=k(D) \# k\left(D^{\prime}\right)$. It follows that the diagrams of prime knots are atoms. We leave as a research topic to investigate the relationships between the unique factorization of a knot and the atomic decompositions of its diagrams.
\end{abstract}

Planar t-graphs.

A $t$-graph $G$ contains more information than the underlying graph $\operatorname{Graph}(G)$ and less information than a map $M$ in $\mathcal{M}_{2,2}^{t}$ such that $\operatorname{Graph}^{t}(M)=G$. Planar graphs and planar maps are characterized by finitely many forbidden minors ([Die, MT] for graphs, [Cou00, CouDus] for maps). Our question is:

Are planar t-graphs characterized by finitely many forbidden substructures of some kind?

We have given a natural characterization of Gauss multiwords (Proposition 25(2)), hence, of Gauss words. However, there exist other characterizations of Gauss words: the one of [LM] uses a notion of forbidden structure on words and the one of [Ros, FOM] uses the interlacement graph associated with a double occurrence word.

Can these characterizations be extended to Gauss multiwords?

Many extensions remain to be investigated. First, intersections of curves on other surfaces than the plane (see [LRS]). Our characterization of Gauss multiwords in terms of the planarity of an associated $t$-graph extends easily to other surfaces. Since the embeddability of a graph in an arbitrary given surface can be checked in linear time by an algorithm that produces embeddings ([Moh]), the algorithm of Proposition 15 can be turned into a linear-time algorithm for checking if a double occurrence multiword is associated with curves in a given surface.

Can one extend to other surfaces the characterization (resulting from Corollary 21 and its consequences) of all tuples of curves in a given surface that correspond to a given double occurrence multiword? 
Some other questions:

Can the results of this article be generalized to the description of curves with multiple intersections?

Gauss multiwords are defined from curves that cross, themselves or pairwise. One could also consider curves that have touching points i.e., that can share a vertex without crossing at it. This notion is used in [FOM]. Special labels in double occurrence multiwords could encode touching points.

What are the associated multiwords and when are they unambiguous? What are the tuples of curves with crossing and touching points associated with a given multiword?

Acknowledgement: I thank E. Gioan and the late M. Las Vergnas for many helpful comments.

\section{References}

[Ada] C. Adams, The knot book, AM.S., 2004.

[Bol] B. Bollobas, Modern graph theory, Springer, 2001.

[ChaWeb] N. Chaves, C. Weber, Plombages de rubans et problème des mots de Gauss, Exp. Math. 12 (1994) 53-77.

[Cou00] B. Courcelle, The monadic second-order logic of graphs XII: Planar graphs and planar maps, Theoret. Comput.Sci. 237 (2000) 1-32.

[CouAD] B. Courcelle, The atomic decomposition of strongly connected graphs, June 2013, submitted for publication. (See http://hal.archives-ouvertes.fr/hal00875661).

[CouDus] B. Courcelle and V.Dussaux, Map genus, forbidden maps and monadic second-order logic, The Electronic Journal of Combinatorics 9 (1) (2002), R40.

(See http://www.combinatorics.org/Volume_9/Abstracts/v9i1r40.html).

[Die] R. Diestel, Graph theory, Springer-Verlag, 4th edition, 2010. (See http://diestel-graph-theory.com).

[FOM] H. de Fraysseix and P. Ossona de Mendez, On a characterization of Gauss codes, Discrete Comput. Geom. 22 (1999) 287-295.

[GodRoy] C. Godsil and G. Royle, Algebraic Graph Theory, Springer, 2001. 
[LRS] S. Lins, B. Richter, H. Shank, The Gauss code problem off the plane. Aequationes Mathematicae 33 (1987) 81-95.

[LM] L. Lovasz and M. Marx, A forbidden substructure characterization of Gauss codes, Bulletin of the AMS 82 (1976) 121-122.

[Moh] B. Mohar, A Linear Time Algorithm for Embedding Graphs in an Arbitrary Surface, SIAM J. Discrete Math. 12 (1999) 6-26.

[MT] B. Mohar and C. Thomassen, Graphs on Surfaces, The Johns Hopkins University Press, 2001.

[Ros] P. Rosenstiehl, Solution algébrique du problème Gauss sur la permutation des points d'intersection d'une ou plusieurs courbes fermées du plan, C.R. Acad. Sc. Paris, Sér. A 283 (1976) 551-553.

[Tut] W. Tutte, Graph Theory, Addison-Wesley, 1984.

\title{
Appendix : Review of some definitions.
}

\author{
Isomorphisms \\ Isomorphism of graphs : notation $G \cong H$, Section 2.1.1, p. 6 \\ v-isomorphism of graphs (identity on vertices) : Section 2.1.1, p. 6 . \\ Automorphisms and v-automorphisms : Section 2.1.1., p. 6 \\ v_isomorphisms of $t$-graphs : Definition 2, Section 2.1.2., p. 6
}

Homeomorphisms

Homeomorphisms that preserve the orientation correspond to the equality of maps; general homeomorphisms correspond to equivalence of maps : Section 2.2, Definition 4,p.8.

Equivalences

Conjugacy of sequences : notation $\equiv$, Section 5.1.1,p.20.

Conjugacy combined with reversal : notation $\asymp$, Section 5.1.1,p.20.

Equivalent maps $\left(M=N\right.$ or $\left.M=N^{-1}\right)$ : Section 2.2, Definition 4,p.8.

Equivalent multiwords (by reversal of some components) : notation $W \asymp W^{\prime}$, Section 5.1.1,p.20.

$t$-equivalent maps (same associated $t$-graph): notation $\sim^{t}$, Definition 5, p.8 (it is implied by equivalence).

Equivalent terms (same value) : Section 3.2, Definition 10. 